\title{
Scaling Up Modulo Scheduling For High-Level Synthesis
}

\author{
Leandro de Souza Rosa*, Christos-Savvas Bouganis ${ }^{\dagger}$, and Vanderlei Bonato* \\ *Institute of Mathematics and Computer Sciences \\ The University of São Paulo - São Carlos, Brazil, \\ Email: \{leandrors, vbonato\}@usp.br \\ †Imperial College London - London, England \\ Email: christos-savvas.bouganis@imperial.ac.uk
}

\begin{abstract}
High-Level Synthesis tools have been increasingly used within the hardware design community to bridge the gap between productivity and the need to design large and complex systems. When targeting heterogeneous systems, where the CPU and the FPGA fabric are both available to perform computations, a design space exploration is usually carried out for deciding which parts of the initial code should be mapped to the FPGA fabric such as the overall system's performance is enhanced by accelerating its computation via dedicated processors. As the targeted systems become more complex and larger, leading to a large design space exploration, the fast estimative of the possible acceleration that can be obtained by mapping certain functionality into the FPGA fabric is of paramount importance. Loop pipelining, which is responsible for the majority of HLS compilation time, is a key optimization towards achieving high-performance acceleration kernels. A new modulo scheduling algorithm is proposed, which reformulates the classical modulo scheduling problem and leads to a reduced number of integer linear problems solved, resulting in large computational savings. Moreover, the proposed approach has a controlled trade-off between solution quality and computation time. Results show the scalability is improved efficiently from quadratic, for the state-of-the-art method, to linear, for the proposed approach, while the optimized loop suffers a $1 \%$ (geomean) increment in the total number of cycles.
\end{abstract}

Index Terms-Loop Pipeline, Modulo Scheduling, High-Level Synthesis, Genetic Algorithm.

\section{INTRODUCTION}

Aiming for high productivity and high-performance hardware designs, many practitioners and researchers rely on High-Level Synthesis (HLS) tools for designing their systems. In many cases, the target systems are heterogeneous, where a CPU and an FPGA fabric are available for the computations. Mapping a complex system in such platforms requires a decision on which parts of the application are best suited for each technology, followed by their actual implementation on the corresponding technology. Currently, for the mapping of the application to the FPGA element, modern HLS tools require user provided directives to guide the compilation mapping process, aiming to increase a performance metric. These directives usually target memory partitioning, loop unrolling, loop tiling, and loop pipelining.

Defining the most appropriated directive settings is a challenging problem, as the optimization of the code that is mapped to the FPGA leads to a combinatorial Design Space Exploration (DSE) problem [1], which is often explored partially by the user over many iterations [2]. Regarding automating the DSE, [3], [1], [4], [5] propose the analysis of many optimization directives, employing heuristics and statistic models to reduce the number of options in the design space, achieving a reduction of $19.5 \times$ on average over brute force search [3], [5].

Complementary to the above approaches, a direct way to reduce the DSE total time is by reducing the time taken to apply each optimization in the code. As loops usually are responsible for the bulk of computation in many applications, loop optimizations are considered to have the most impact on performance and as such, loop pipelining is a key optimization to achieve high throughput. However, most of the HLS compilation time is taken by the modulo scheduling algorithms used to create the loop pipelines [6], restricting as such the efficient DSE in systems that contain loops with a large number of instructions.

This work proposes a new modulo scheduling problem formulation that achieves a better asymptotic complexity with the number of instructions in the loop body than the state-of-the-art approaches. Additionally, the new presented methodology allows to control the balance between time available for the problem solution and the achieved quality of results, enabling fast exploration at the initial DSE stages and higher quality designs in the final stages.

This paper is structured as follows: Section II presents the modulo scheduling problem, and Section III presents the state-of-the-art modulo schedulers in literature. Section IV presents the new formulation proposed for the modulo scheduling problem that creates an explicit separation between the scheduling and allocation parts, exploring the last with a Genetic Algorithm (GA) presented in section V. Section VI presents a theoretical comparison and results over benchmarks between the proposed and state-of-the art schedulers. Finally, Section VII concludes the paper. 


\section{Modulo Scheduling Problem}

The modulo scheduling problem is defined as given a piece of code that contains a loop, the aim is to find the minimum possible initiation interval $I I$ (i.e after how many clock cycles a new loop iteration can be started), a schedule of the loop instructions, and an allocation into the available resources, without violating any dependencies.

An example of the above problem is captured in Code 1, which also introduces the basic modulo scheduling concepts. The number of loop iterations is captured by the loop trip count $t c$ that is defined as $t c=U B-L B+1$. Each instruction $i$ has an associated delay $D_{i}$ representing the number of cycles for its execution.

Code 1: loop code example.

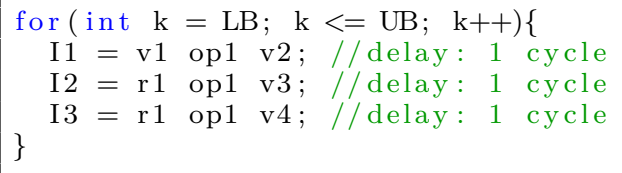

The loop latency $l$ is defined as the number of clock cycles that one loop iteration takes to complete. The latency $l$ is calculated considering a single loop iteration execution as $l=\max \left(t_{i}+D_{i}\right)$, where $t_{i}$ is the cycle where instruction $i$ starts its execution (referred as "starting time"). The loop latency $l$ depends on the number and type of the available resources and on the instructions' schedule. Table I presents four possible schedules for Code 1, assuming a single type of resource (op1), where $I_{i}^{j}$ represents the execution of instruction $I_{i}$ on iteration $j$, each row represents a clock cycle and each column a resource instance.

In Table I, the first two schedules are not pipelined. Thus, all instructions in a loop iteration are scheduled to be completed before the next loop iteration starts. The first schedule uses only one resource, resulting in $l=3$, while the second one uses two resources and has $l=2$. A loop without pipeline takes $t_{\text {otal }} l_{\text {cycles }}=t c \times l$ clock cycles to complete its execution.

The last two schedules represent cases where pipelining has been performed and two and three resources are available respectively. Using two resources, $I_{2}^{k}$ can start its execution together with $I_{3}^{k}$, thus $I I=2$. Analogously, with three resources, $I_{1}^{k+1}$ can start its execution together with $I_{2}^{k}$ and $I_{3}^{k}$, thus $I I=1$. A pipelined loop finishes an iteration every $I I$ cycles, except for the first one that takes $l$ cycles, resulting in total cycles $=l+I I \times(t c-1)$ clock cycles to finish its execution.

TABLE I: Four possible schedule for Code 1.

\begin{tabular}{c||c||c|c||c|c||c|c|c}
\multicolumn{1}{l||}{} & \multicolumn{3}{c||}{ no pipeline } & \multicolumn{5}{c}{ pipeline } \\
\hline cycle & 1 res & \multicolumn{2}{c||}{2 res } & \multicolumn{2}{c}{2 res } & \multicolumn{3}{c}{3 res } \\
\hline 0 & $I_{1}^{0}$ & $I_{1}^{0}$ & - & $I_{1}^{0}$ & - & $I_{1}^{0}$ & - & - \\
\hline 1 & $I_{2}^{0}$ & $I_{2}^{0}$ & $I_{3}^{0}$ & $I_{2}^{0}$ & $I_{3}^{0}$ & $I_{1}^{1}$ & $I_{2}^{0}$ & $I_{3}^{0}$ \\
\hline 2 & $I_{3}^{0}$ & $I_{1}^{1}$ & - & $I_{1}^{1}$ & - & $I_{1}^{2}$ & $I_{2}^{1}$ & $I_{3}^{1}$ \\
\hline 3 & $I_{1}^{1}$ & $I_{2}^{1}$ & $I_{3}^{1}$ & $I_{2}^{1}$ & $I_{3}^{1}$ & $I_{1}^{3}$ & $I_{2}^{2}$ & $I_{3}^{2}$ \\
\hline 4 & $I_{2}^{1}$ & $I_{1}^{2}$ & - & $I_{1}^{2}$ & - & $I_{1}^{4}$ & $I_{2}^{3}$ & $I_{3}^{3}$ \\
\hline 5 & $I_{3}^{1}$ & $I_{2}^{2}$ & $I_{3}^{2}$ & $I_{2}^{2}$ & $I_{3}^{2}$ & $I_{1}^{5}$ & $I_{2}^{4}$ & $I_{3}^{4}$
\end{tabular}

Current approaches for solving the module scheduling problem, and finding the minimum $I I$ that results to the fastest loop execution, rely on first trying to find a solution for an estimating lower bound for $I I$, and in case they fail, to iteratively increase the target $I I$ and try again till a solution is found. The lower bound for $I I$ is defined as $M I I=\max (\operatorname{Rec} M I I, \operatorname{Res} M I I)$, where RecMII is the recurrence minimum $I I$, and ResMII is the resourceconstrained minimum $I I[6]$. Thus, given a candidate $I I$, the problem of minimising total cycles $_{\text {can }}$ be expressed as finding a schedule for the target candidate $I I$ with minimum latency $l$, which can be obtained through an As Soon As Possible (ASAP) schedule.

In this paper, we divide the modulo scheduling problem into its scheduling and allocation parts. The scheduling part consists of defining the starting time $t_{i}$ for all instructions $I_{i}$ in the loop such that no dependencies are violated. The allocation part consists of choosing which congruence class and resource instance (defined as variables $m_{i}$ and $r_{i}$ respectively) instruction $I_{i}$ will be executed. Note that the congruence class $m_{i}$ of instruction $I_{i}$ is defined as $m_{i}=t_{i} \% I I$. The above information is represented through a Module Reservation Table (MRT), which is a $I I \times \sum_{k \in R} a_{k}$ matrix, where $a_{k}$ is the instances number of resource type $k$ and $R$ is the set of all resource types.

Through the rest of this paper, we will consider the modulo scheduling problem for a given $I I$, assuming there is an outer loop searching for the possible $I I$, as is the case in all state-of-the-art approaches.

\section{State-of-the-ARt Modulo Scheduling APPROACHES}

\section{A. SDC Modulo Scheduler}

Zhang et. al. [7] proposed SDCS, an approach that divides the modulo scheduling problem into its scheduling and allocation parts. The scheduling part includes the dependency, timing, and chaining constraints captured through a System of Difference Constraints (SDC) form. As such, a Totally Uni-Modular (TUM) matrix of constraints can be constructed, whose linear relaxation solution is integral, and thus is optimal for the original problem as well [8]. As such, the original integer-programming problem, with exponential solve complexity, is mapped to its relaxed version that exhibits polynomial solve complexity.

Definition: An MRT (an allocation) is said "valid" if all positions (slots) are assigned to up to one instruction

The allocation part is solved with a greedy heuristic, which starts solving the scheduling problem without considering the resource constraints. If the resulting MRT is not valid, the heuristic adds new SDC constraints to the SDC problem, in an attempt to force the conflicting instructions to start at different times. The process is repeated until there are no conflicts in the MRT, or until a certain number of attempts are performed. If the algorithm fails to find a valid allocation, the candidate $I I$ is increased, and the whole process is repeated.

The above iterative heuristic approach [7] is improved in [6], by introducing the possibility of backtracking on 
the conflict instructions, allowing them to be sent back to the scheduling queue, significantly improving the solutions latency and also finding solutions for $I I$ candidates for which the previous approach would have failed. However, the number of iterations of the method is increased, and consequently the number of SDC problems solved is also increased.

The example below illustrates the number of SDC problems that are required to be solved by this approach in the worst-case. The scheduler (Algorithm 1) and backtracking (Algorithm 2) algorithms are replicated from [6].

Definition: "loop size" is defined as the number of instructions in the Intermediate Representation (IR) code of the loop body. "problem size" is defined as the number of variables and constraints in an optimization problem formulation.

Firstly, in Algorithm 1, the non-resource constrained schedule is calculated once, resulting in the As Soon As Possible (ASAP) schedule (line 1) that serves as the basis for setting the instructions starting time. In lines 2 to 4 , the instructions are queued to be processed in order. The number of iterations is constrained by the budget (line 3 ), which is defined as budget $=$ budgetRatio $* n$, where $n$ is the loop size and budgetRatio is a user defined constant. As such, the above limit makes the worst-case number iterations of Algorithm 1 equal to budget, regardless on how many instructions are sent back to the scheduling queue by the backtracking policy. In each iteration, the solver, i.e. the function responsible for solving the SDC scheduling problem, is invoked in lines 11 and 17 as well as a number of times within the BackTracking function.

As illustrated in the BackTracking function (Algorithm 2 ), an instruction $I$ is attempted to be scheduled from its ASAP time to its current scheduled time (lines 1 to 3 ). In the worst-case, its ASAP time is zero and its current scheduled time is the length of the whole schedule, which, in the worst-case, is a sequential path. Therefore, in the worst-case, the solver is invoked $\sum_{k=0}^{n} D_{k}$ times, where $D_{k}$ is the delay of instruction $k$. Assuming that all instructions have a maximum latency $D_{\max }$, it results in $n \times D_{\max }$ solver invocations in the backtracking function.

As such, in the worst-case, the total number of solver calls is:

$$
\begin{gathered}
\mathcal{O}\left(\text { budgetRatio } \times n\left(2+n \times D_{\max }\right)\right)=\ldots \\
\ldots=\mathcal{O}\left(\text { budgetRatio } \times n^{2}\right)
\end{gathered}
$$

The rest of Algorithm 2 deals with the case where the provided schedule is not feasible. In that case, a time slot is selected (lines 4 to 8 ) and an instruction from that slot is removed from the SDC problem formulation (lines 9 to 12) and the necessary updates are performed in the corresponding MRT structure (lines 13 to 17). As such, a time slot is available for an instruction to be scheduled (lines 18 and 19), and the control returns to Algorithm 1.

\section{B. ILP Modulo Scheduler}

As the objective is to create a schedule for the loop instructions for a given $I I$, the problem can be naturally

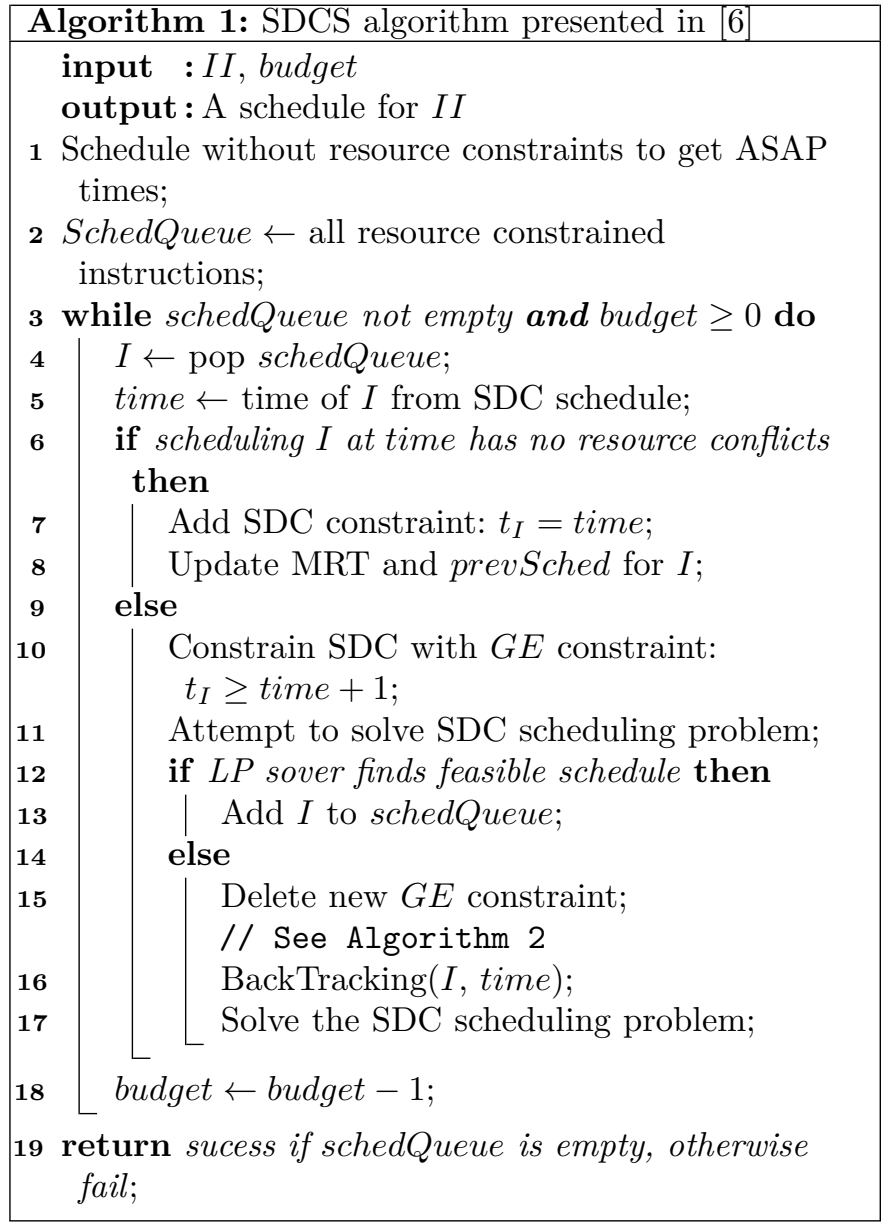

formulated as a resource-constrained scheduling-allocation Integer Linear Problem (ILP). Additionally to resources, other HLS metrics can be added in the ILP to improve or ensure hardware quality, as timing, chaining [8], and soft [9] constraints.

Instead of separating the problem scheduling and allocation parts, [10] proposes to use the complete formulation, which always allows finding the optimal solution for a given $I I$, if it exists. This formulation requires $\mathcal{O}\left(n^{2}\right)$ variables and constraints to ensure MRT validity, which arecalled overlap variables [11], where $n$ is the loop size. Moreover, as this approach cannot be expressed in the SDC form, its solver scales exponentially with the problem size. However, there is no need to solve the formulation multiple times as in the SDCS approach but only once, which counterbalances the computation time required for obtaining a single solution. Moreover, the ILP approach is also capable to quickly prove that the problem generated by a candidate $I I$ is infeasible, which is a secondary way to counter-balance its exponential complexity. We will refer to it by ILP Scheduler (ILPS) in this work.

Formulation 1 presents a simplified version of the ILP formulation presented in [10], where the variables domain is described in Table II (replicated from [10]).

The objective function (line 2) captures the starting time of all instruction, which needs to be minimized, leading to 


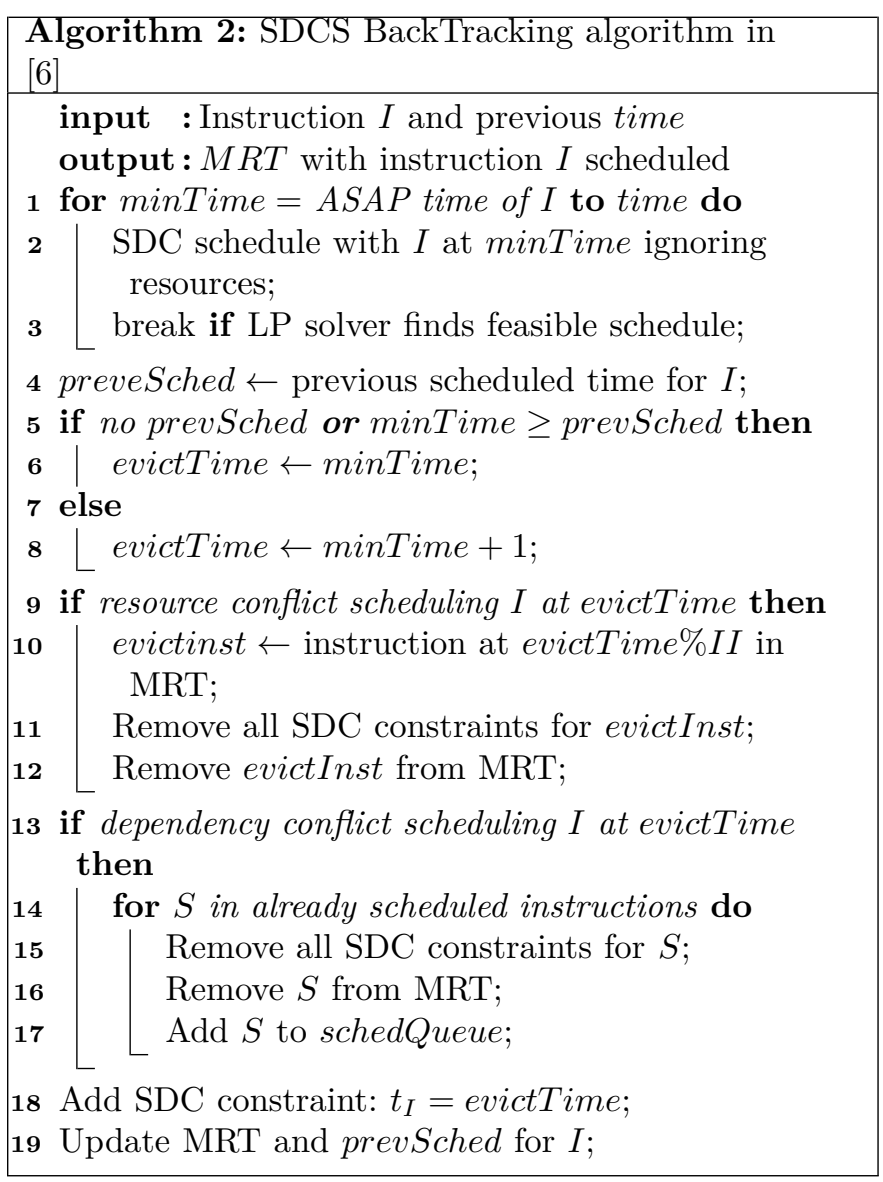

an ASAP schedule.

For each dependency on the data flow, a constraint is created (line 4 ), where $b_{i j} \geq 0$ is the loop-carried dependency distance (which is represented as a back-edge in the data-flow graph), and $b_{i j}=0$ for data dependencies (forward edges in the data flow). Note that [10] considers $b_{i j} \in\{0,1\}$, while this paper adopts the more general case where $b_{i j} \in \mathbb{Z}_{0}^{+}$, as proposed by [6].

Formulation 1: Simplified version of ILP formulation for modulo scheduling presented in [10]

$$
\begin{array}{ll}
\underset{\sum_{i \in O} t_{i}}{\operatorname{minimize}} & \\
\text { subject to: } & \leq t_{j}+b_{i j} I I \\
t_{i}+D_{i} & \leq 1 \\
\epsilon_{i j}+\epsilon_{j i} & \geq 0 \\
r_{j}-r_{i}-1-\left(\epsilon_{i j}-1\right) a_{k} & \leq 0 \\
r_{j}-r_{i}-\epsilon_{i j} a_{k} & \leq 1 \\
\mu_{i j}+\mu_{j i} & \geq 0 \\
m_{j}-m_{i}-1-\left(\mu_{i j}-1\right) I I & \leq 0 \\
m_{j}-m_{i}-\mu_{i j} I I & \geq 1 \\
\epsilon_{i j}+\epsilon_{j i}+\mu_{i j}+\mu_{j i} & =y_{i} I I+m_{i} \\
t_{i} & \leq a_{k}-1 \\
r_{i} & \leq I I-1 \\
m_{i} & \leq S L_{\max } \\
t_{i}+D_{i} &
\end{array}
$$

To guarantee that two instructions will not occupy the
TABLE II: Problem signature from the ILP modulo scheduling replicated from [10]

$\begin{array}{ll}\text { Input } & \\ O I & \text { Initiation Interval; } \\ \left.D_{i}, i \in O, \ldots, n-1\right\} & \text { IR Instructions; } \\ E=\{(i \rightarrow j ; b)\} \subseteq O \times O \times \mathbb{Z}_{0}^{+} & \text {Instruction } i \text { delay; } \\ & \text { Dependency edges on } \\ R=m e m, d s p & \text { datapath. } b \geq 1 \Leftrightarrow \text { backedge; } \\ A=a_{k} \mid k \in R & \text { Resource types; } \\ L_{k} \subseteq O & \text { Instance of resource } k \\ L=U_{k \in R} L_{k} & \text { Resource limited operations of } \\ & \text { type } k ; \\ \text { Output } & \text { Union of all resource limited } \\ t_{i}, i \in O & \text { operations; } \\ r_{i}, i \in L & \text { Start time of operation } i \\ \text { Decision Variables } & \text { Resource instance used by } \\ t_{i}, i \in O & \text { operation } i ; \\ r_{i}, i \in L & \\ m_{i}, i \in L & \text { Start time of operation } i ; \\ y_{i}, i \in L & \text { Resource instance used by } \\ \epsilon_{i j}, \forall k \in R: \forall i, j \in L_{k}, i \neq j & \text { operation } i \\ \mu_{i j}, \forall k \in R: \forall i, j \in L_{k}, i \neq j & \text { Congruence class of operation } \\ & i ; \\ & \text { Helper variable of operation } i \\ & =1 \text { if } r_{i}<r_{j},=0 \text { otherwise; } \\ m_{i}<m_{j},=0\end{array}$

same MRT slot, the binary overlap variables $\epsilon_{i j}$ (lines 5 to 7 , and 11) and $\mu_{i j}$ (lines 8 to 11) are defined for each pair of instructions $I_{i}$ and $I_{j}$ that share the same resource type $k$, making the number of overlap variables to grow quadratically with the loop size. Lines 4 and 15 capture the dependency and timing constraints used in [10]. Lines 13 and 14 provide the upper bounds for $r_{i}$ and $m_{i}$, respectively. Line 12 links the values of $t_{i}$ to $m_{i}$ using the auxiliary variables $y_{i}$. We use the notation on Table II through the remaining of this paper.

[12] presents a comparison between the existing module schedulers in the literature, including iterative, slack, swing and stage modulo schedulers, that concludes that the iterative modulo scheduler (which is the base for SDCS) achieves better $I I$ values, despite requiring longer executions times. As the achieved value of $I I$ is critical in the HLS context when targeting high-performance designs, the iterative method has been chosen to act as a baseline in this work.

\section{SDC Formulation for Separating Scheduling AND Allocation}

The main idea behind the proposed approach is to separate the ILP formulation scheduling and allocation parts, and to use an SDC formulation to solve the scheduling part and another method to explicitly traverse through valid MRTs (i.e. the allocation space). This approach differs from [6], which modifies the SDC problem (scheduling part) by adding constraints trying to force its solution to have a valid MRT indirectly.

Section IV-A presents how Formulation 1 is transformed to allow the scheduling and allocation parts to be separately explored. Section IV-B presents a relaxation of the 
proposed formulation, that is useful due to its feasibility properties.

\section{A. Separating Scheduling and Allocation}

On Formulation 1, the overlap variables (lines 5 to 11 ) guarantee that two instructions are not allocated to the same MRT slot, and thus ensuring a valid MRT. In the proposed approach, the allocation part will ensure a valid MRT construction, and as such the overlap variables are eliminated from the formulation. The rest of the problem formulation has: dependency constraints (line 4), other HLS constraints (line 15), and the linker constrains between $t_{i}$ and $m_{i}$ (line 12). As such, the remaining problem can be rewritten as:

$$
\begin{array}{ll}
\text { minimize: } & \sum_{i \in O} t_{i} \\
\text { subject to: } & \\
t_{i}-t_{j} & \leq b_{i j} I I-D_{i} \\
t_{i} & =m_{i}+y_{i} I I
\end{array}
$$

Substituting $t_{i}$ by $m_{i}+y_{i} \times I I$ in all constraints and objective function, $t_{i}$ is eliminated from the problem, and $y_{i}$ is promoted to a decision variable, resulting in Formulation 2 . Note that $t_{i}$ values can be calculated with the $m_{i}$ values and the solution $y_{i}$.

Formulation 2: SDC base problem for scheduling and allocation separation

$$
\begin{aligned}
& \text { minimize: } \quad \sum_{i \in O} y_{i} * I I+m_{i} \\
& \text { subject to: } \\
& y_{i}-y_{j} \\
& \leq\left\lfloor\frac{b_{i j} I I-D_{I}-\left(m_{i}-m_{j}\right)}{I I}\right\rfloor
\end{aligned}
$$

Other HLS constraints in the SDC can also be added in Formulation 2 in a similar way.

Assuming a valid MRT has been produced by the allocation stage, the $m_{i}$ and $r_{i}$ are known, and by solving the SDC Formulation 2, the $y_{i}$ values are calculated in polynomial time, leading to the $t_{i}$ values. Furthermore, the latency of the solution is used as an MRT measure of quality, since solving Formulation 2 for different MRTs results in schedules that differ only in latency, which is the only non-constant factor in total $_{\text {cycles }}=l+I I \times(t c-1)$, for a given $I I$.

Summarising, formulation 2 allows us to divide the modulo scheduling problem into the allocation and scheduling parts explicitly. The proposed method traverses the MRT space and produces possible allocations, which then drive the scheduling part through the SDC Formulation 2. The traversing of the MRT space is guided by the latency of the obtained solutions.

The traversing of the MRT space can be performed by various methods such as any heuristic, meta-heuristic, evolutionary algorithm, machine learning techniques, while the scheduling part can be optimally solved using Formulation 2 , in polynomial time.

Nevertheless, Formulation 2 is not feasible for any MRT construction.
Definition: We define "feasible MRT" and "infeasible MRT" as MRTs to which Formulation 2 is feasible and infeasible, respectively.

The feasibility and infeasibility of Formulation 2 is demonstrated by the example in Figure 1a. Let's assume that we only have 2 resource instances to execute instructions $I_{i}=\{1,2,3,4,5\}$, thus $\min I I=3$ and the $M R T$ has 3 rows and 2 columns. Figure $1 \mathrm{~b}$ and $1 \mathrm{e}$ show the NonResource Constrained As Soon As Possible (NRCASAP, and the optimal MRTs, respectively.

Figure 1c shows a feasible MRT, with scheduling times $\mathbf{t}=\{0,1,1,3,2\}$, and the back-edge constraint given as $t_{5}-t_{1} \leq b_{5,1} \times I I-D_{5} \Rightarrow t_{1} \geq 0$, which is satisfied since $t_{1}=0$. Figure $1 \mathrm{~d}$ shows an infeasible MRT, with scheduling times $\mathbf{t}=\{0,3,1,1,5\}$, and the back-edge constraint given as $t_{5}-t_{1} \leq b_{5,1} \times I I-D_{3} \Rightarrow t_{1} \geq 3$, which is not satisfied since $t_{1}=0$.

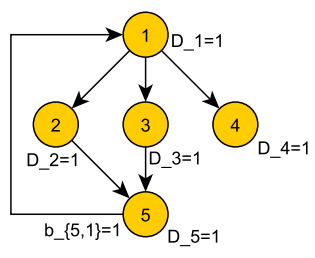

(a) Data flow

\begin{tabular}{c|c|c}
$\mathrm{m}$ & $a_{0}^{0}$ & $a_{0}^{1}$ \\
\hline 0 & $i_{1}$ & $i_{4}$ \\
\hline 1 & $i_{2}$ & $i_{3}$ \\
\hline 2 & $i_{5}$ & -
\end{tabular}

(c) feasible MRT

\begin{tabular}{c|c|c}
$\mathrm{m}$ & $a_{0}^{0}$ & $a_{0}^{1}$ \\
\hline 0 & $i_{1}$ & $i_{2}$ \\
\hline 1 & $i_{4}$ & $i_{3}$ \\
\hline 2 & $i_{5}$ & -
\end{tabular}

(d) infeasible MRT

\begin{tabular}{c|c|c}
$\mathrm{m}$ & $a_{0}^{0}$ & $a_{0}^{1}$ \\
\hline 0 & $i_{1}$ & - \\
\hline 1 & $i_{2}$ & $i_{3}$ \\
\hline 2 & $i_{5}$ & $i_{4}$
\end{tabular}

(e) optimal MRT
Fig. 1: Example of different MRTs for a DFG.

As such, the allocation space exploration needs to also consider infeasible MRTs, for which the quality of the obtained solution (i.e. latency) is not available. As the MRT is infeasible, the operations cannot be scheduled, and as such, there is no quality metric (i.e. schedule latency) associated with the MRT. In this fashion, a possible solution is to discard the infeasible MRTs and keep generating MRTs and analysing only the feasible ones, as summarized in the flow presented in Figure 2. However, experimental work indicated that finding a feasible MRT through the above algorithm is rare. More specifically, using benchmark cp (described in Section VI-C), 145872.23 random MRTs on average (50 repetitions) need to be created before finding a feasible one.

Instead of discarding infeasible MRTs, this paper proposes an approach that would allow the association of an infeasible MRT with a latency value, and as such would allow its incorporation in the MRT exploration stage. The proposed approach is based on relaxing Formulation 2. The relaxed formulation solution results in a schedule for the loop with an associated latency, even though this may not be an actual solution for the non-relaxed problem. However, 


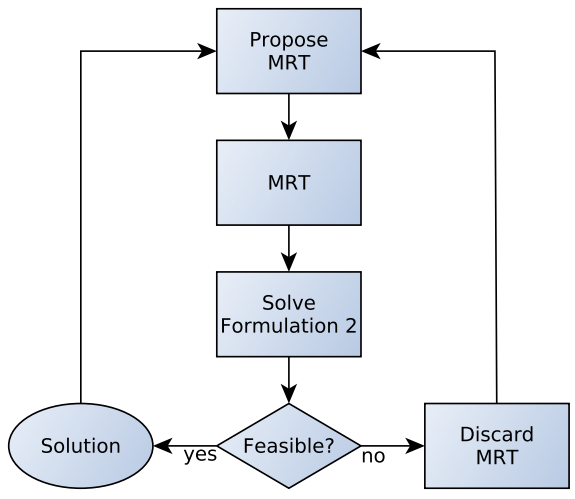

Fig. 2: Straight forward flow to handle infeasible MRTs.

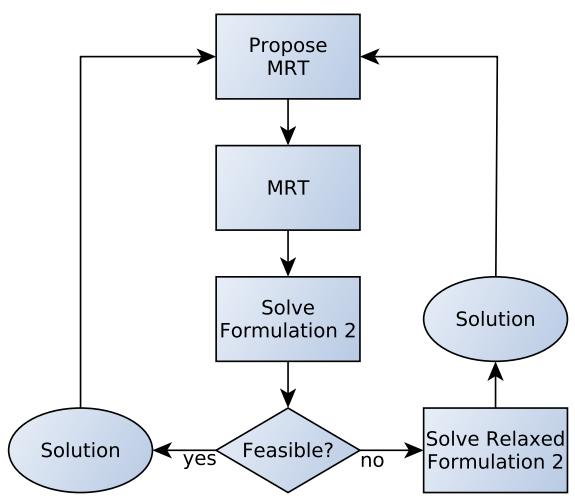

Fig. 3: Flow to handle infeasible MRTs using relaxation of Formulation 2.

it can be used to compare infeasible MRTs with feasible ones, as illustrated in the flow in Figure 3.

Section IV-B presents a relaxation for Formulation 2 that is guaranteed to be feasible even for infeasible MRTs.

\section{B. A Relaxation of Formulation 2}

Formulation 3 is a relaxation of Formulation 2 by renaming $y_{i} I I=x_{i}$.

Formulation 3: SDC base problem relaxation for scheduling and allocation separation

$$
\begin{aligned}
& \operatorname{minimize:} \\
& \sum_{i \in O} x_{i}+m_{i} \\
& \text { subject to: } \leq-D_{i}+b_{i j} I I-\left(m_{i}-m_{j}\right) \\
& x_{i}-x_{j}
\end{aligned}
$$

In Formulation 3, there is no guarantee that $x_{i}$ values are multiples of $I I$, and as such, $y_{i}$ are not guaranteed to be integer. Thus, there is no guarantee that $t_{i} \% I I=\left(x_{i}+\right.$ $\left.m_{i}\right) \% I I=m_{i}$. In other words, the MRT that corresponds to the solution (calculated as $t_{i} \% I I$ ) is not guaranteed to be the same valid MRT used to construct the problem in Formulation 3, thus, it is not guaranteed to be valid. This has no consequences for the flow proposed in Figure 3, since the schedule values $\left(t_{i}\right)$ are only used for comparison.
In the following paragraphs, it is proved that if a feasible MRT exists for Formulation 2, then Formulation 3 is always feasible (regardless of the MRT). As such, by employing Formulation 3, the proposed methodology ensures that a schedule can be derived that can be used to guide the DSE process.

Notation: let $\delta x_{a b}$ represent the $x_{a}-x_{b}$.

For the following proof, the following bounds need to be introduced:

1) $-(I I-1) \leq \delta m_{i j} \leq(I I-1)$

2) $0 \leq D_{i} \leq(I I-1)$

3) $0 \leq(I I-1)-D_{i} \leq(I I-1)$

Bound 1 is a consequence that $0 \leq m_{i} \leq(I I-1)$ for any instruction $I_{i}$ by definition. Bound $\mathbf{2}$ implies that instruction $I_{i}$ delay is smaller than $I I$, which is true for functional units are not pipelined themselves. Thus, it is imperative that every instruction has to finish its execution before being executed again. Bound $\mathbf{3}$ is a consequence of bound 2 .

The first consideration to make is that the dependencies created by the forward edges $\left(I_{i} \rightarrow I_{j}\right)$ can always be satisfied regardless of the MRT. This is because the RHS in Formulation 2 can be only 0 or -1 , since $b_{i j}=0$, and bounds 1 and 2 make $-(I I-1)-(I I-1) \leq-D_{i}-$ $\delta m_{i j} \leq 0+(I I-1)$. The forward edge constraints are also always valid for Formulation 3 since it is a relaxation of Formulation 2. Thus, we will only consider the back-edges constraints from now on.

Using bound 1, we can expand the RHS of Formulation 2 to enclose all possible values of the MRT, including the ones that would make it infeasible, resulting in Inequality inq.1.

$$
\left.\delta y_{i j} \leq\left\lfloor\frac{b_{i j} I I-D_{i}-\delta m_{i j}}{I I}\right\rfloor \leq\left\lfloor\frac{b_{i j} I I-D_{i}+(I I-1)}{I I}\right\rfloor\right\rfloor_{\text {inq. } 1)}
$$

In the same fashion, for Formulation 3 to be feasible for any MRT, is equivalent to rewrite the RHS on Formulation 3 as Inequality inq.2. That is, $\delta x_{i j}$ has to be smaller than the lower bound of $b_{i j} I I-D_{i}-\delta m_{i j}$.

$$
\delta x_{i j} \leq b_{i j} I I-D_{i}-(I I-1) \leq b_{i j} I I-D_{i}-\delta m_{i j} \text { (inq.2) }
$$

Thus, the condition for the feasible space of Formulation 3 to encompass all values of MRT is if Inequality inq. 1 is encompassed by Inequality inq.2, resulting in Inequality inq.3.

$$
\left\lfloor\frac{b_{i j} I I-D_{i}+(I I-1)}{I I}\right\rfloor \leq b_{i j} I I-D_{i}-(I I-1) \text { (inq.3) }
$$

If $I I=1$, Inequality inq.3 always holds. Thus, we only need to analyse the case when $I I>1$.

Using bound $\mathbf{3}$, we can see that the Left-Hand-Side (LHS) of Inequality inq. 3 is equal to $b_{i j}$, implying that the condition in Inequality inq. 3 can be rewritten as Inequality inq. 4 . 


$$
\begin{aligned}
b_{i j} & \leq b_{i j} I I-D_{i}-(I I-1) \\
\Rightarrow b_{i j} & \leq b_{i j}+b_{i j}(I I-1)-D_{i}-(I I-1) \\
\Rightarrow b_{i j} & \geq\left\lfloor\frac{D_{i}+(I I-1)}{(I I-1)}\right\rfloor
\end{aligned}
$$

Finally, using bound $\mathbf{3}$, and that $b_{i j} \geq 1$ by definition, we conclude that Inequality inq. 4 always holds, meaning also that Inequality inq. 3 always holds.

As such, if Formulation 2 is feasible for an MRT, then Formulation 3 is always feasible regardless the MRT.

\section{SDC Based Genetic Algorithm}

As noted in Section IV-A, any method of choice can explore the allocation space as long valid and feasible MRTs are produced by the method where Formulation 2 is used to solve the scheduling problem. In this work a Genetic Algorithm (GA) is selected as it is known to be applicable for problems without information about the solution structure [13].

The first step in the creation of a GA is the design of the GA chromosomes. Formulations 2 and 3 allow us to select the MRTs as chromosomes (individuals). In this way, we reduce the number of problem variables (described in lines 5 to 11 on Formulation 1) by capturing these constraints in the GA chromosome encoding. As will be explained later, the chromosome evolution guarantees a valid MRT, and as such, lines 5 to 11 are removed (with the overlap variables).

\section{A. The Genetic Algorithm}

The GA purpose is to evolve MRTs (individuals) according to the final schedule latency (fitness). That is, since $I I$ is given, and $t c$ is constant, the GA evolves individuals to reduce the solution latency. MRTs are implemented as lists of $\left(m_{i}, r_{i}, I_{i}\right)$ triplet, where $\left(m_{i}, r_{i}\right)$ correspond instruction $I_{i}$ MRT slot, which is a common way to represent sparse matrices such as the MRT.

1) Main Algorithm: The proposed GA algorithm is described in Algorithm 3, to which we will refer as GAS. The parameters impacting GAS are the population size $n P o p$, the insemination rate $i_{r}$, the number of new individuals created at each generation offspringSize, the minimum number of generation $\min G e n$, and the mutation probability mutationProb.

The first step is the creation of a random population (lines 1 to 4 ). The initial population is inseminated with individuals created by randomly legalizing the NRCASAP schedule MRT (lines 5 and 11) using the legalization process (described in details in Algorithm 5). Since the NRCASAP schedule is the unreachable ideal, we expect that its $M R T$ values contain "good genes" and the optimality will be close to a slight modification of its $M R T$.

The population insemination introduces some knowledge about the solution structure into the GA, which is shown to improve GA results as shown in [14]. Figure 4 shows the number of generations a typical GAS execution takes to find a feasible MRT in function of the population size, with and without the insemination. For Figure 4, we used the benchmark complex (described in details in Section VI-C).

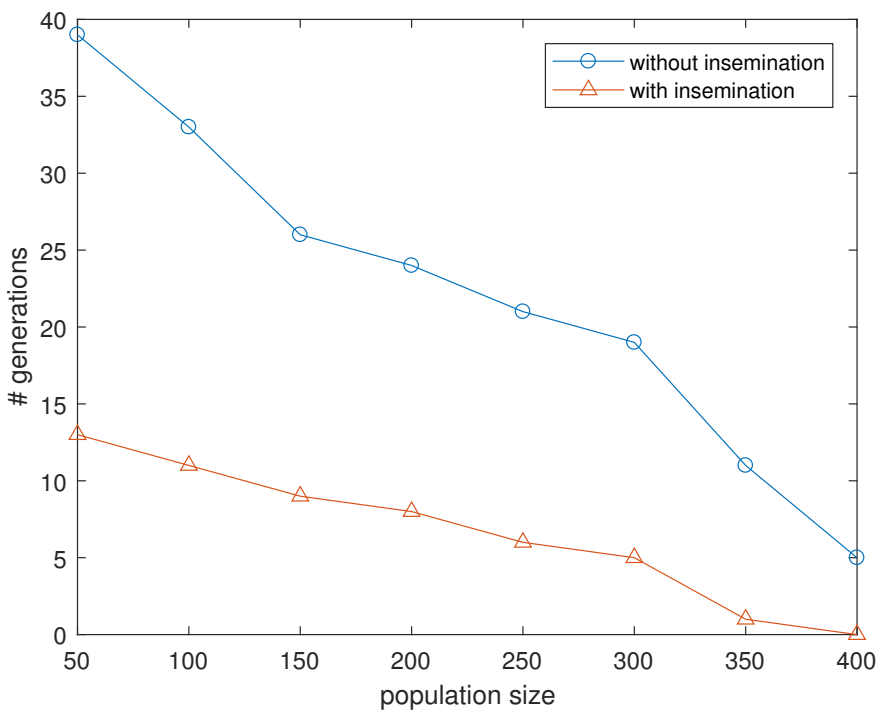

Fig. 4: Average number of generations needed to find a feasible MRT with and without the insemination process.

Consider the example in Figure 1a, with $a_{0}=2$ resource instances and $M I I=3$. Figure $1 \mathrm{~b}$ presents the NRCASAP MRT, which had three instruction with congruence class $m=1$. The legalization process can generate the MRTs presented in Figures 1c, 1d, or 1e, which will be used to inseminate the initial population.

The population is then evolved for minGen generations (lines 13 to 23). The evolution process consists of creating offspringSize new individuals, called cubs, (lines 14 to 22 ), from two different individuals randomly selected from the population (line 15) through a cross-over process (described in details in Algorithm 6). The mutation occurs by attributing a new random empty MRT slot to the instructions in the new created MRTs (lines 18 to 20). Then, the cubs are added to the population (lines 21 and 22 ), what results in a $n P o p+o f f$ springSize individuals population. Selection is made by ordering and selecting the offspringSize best population individuals (lines 23 and 24).

Finally, the algorithm does not stop until it reaches the minimum number of generations minGen, or until it finds a feasible MRT (line 27). Furthermore, we define budget as a maximum number of generations in case a feasible MRT is not found (lines 25 and 26), which cause GAS to return a failure for the given $I I$ meaning that the candidate $I I$ should be increased.

2) Evaluation Algorithm: Algorithm 4 describes the evaluation function, which considers two cases. The first case is when the MRT is feasible, and thus we have a solution for the problem. The second case is when the MRT is infeasible, and thus Formulation 3 is used to calculate the fitness with a penalty given by how many conflicts there were in the solution of Formulation 3.

First, the algorithm tries to solve Formulation 2 (line 1 ), and the resulting schedule latency is returned if it is 


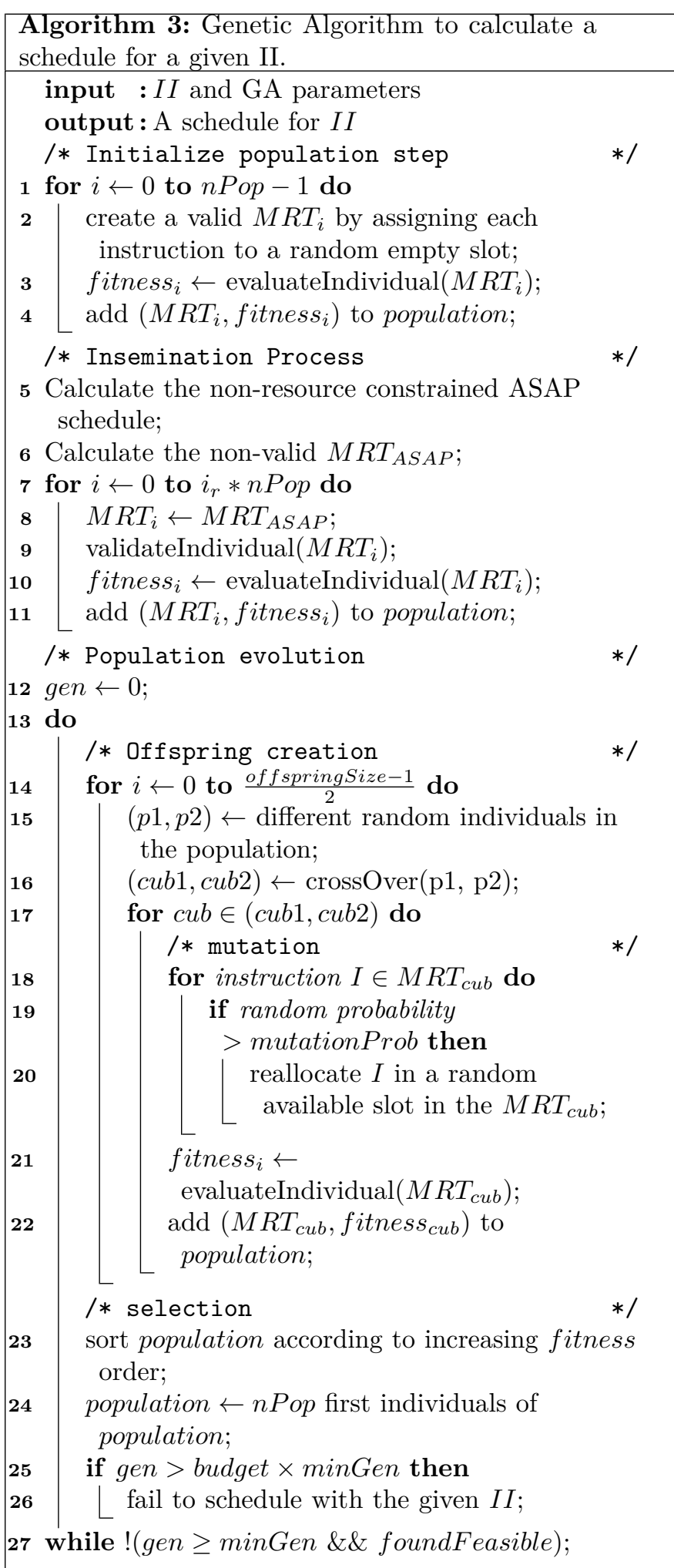

feasible (lines 2 to 5 ). If Formulation 2 is infeasible for the given $M R T$, Formulation 3 (line 7) is solved, which is guaranteed to be feasible (Section IV-A). The MRT corresponding to the relaxed problem solution $M R T_{\text {relaxed }}$ is calculated using $m_{i}^{\text {relaxed }}=t_{i} \% I I$ (line 9 ), where $t_{i}=$ $\left(x_{i}+m_{i}\right)$. The latency is calculated using $t_{i}$ values (line $9)$. Since $M R T_{\text {relaxed }}$ is not guaranteed to be valid, we modify it using the validation function (line 11), which return how many resource conflicts the non-valid $M R T$ had and randomly fixes the $M R T$ (described in Algorithm $5)$. Finally, the function returns the latency $l$ for the relaxed schedule plus a penalty according to the number of resource conflicts the MRT had times II (line 12). This penalty captures that the relaxed schedule can be a solution for the non-relaxed one, which happens when no resource conflicts occur, and also punishes relaxed schedules with conflicts, making them less likeable to survive through generations.

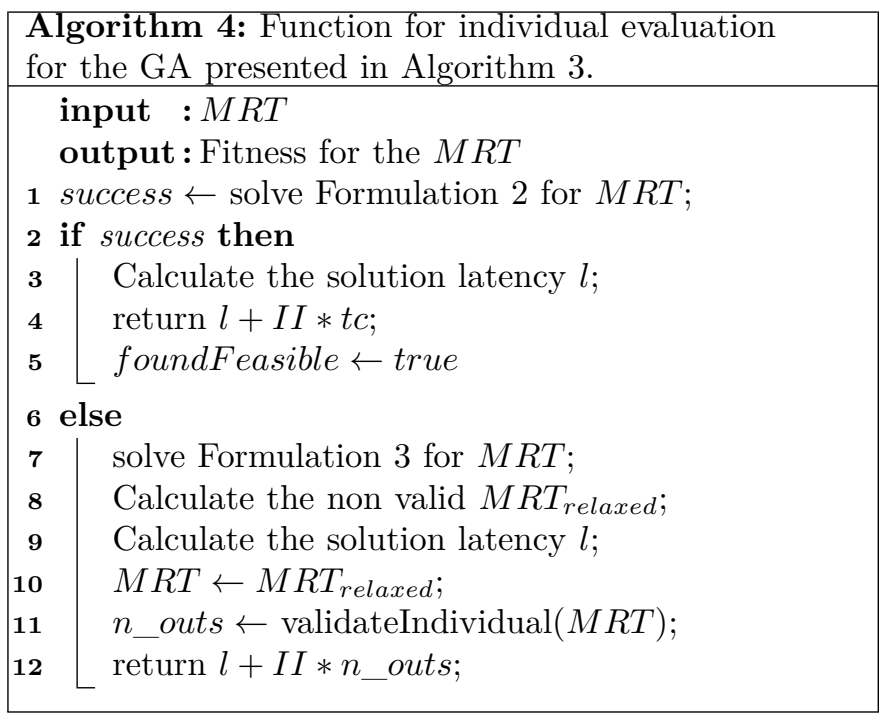

As example, consider Figure 1c, which is feasible and Formulation 2 returns the schedule $\mathbf{t}_{1 c}=\{0,1,1,2,3\}$, with latency $l_{1 c}=4$, resulting in fitness $s_{1 c}=4$.

Now, consider Figure 1d, which is infeasible, and solving Formulation 3 results in $\mathbf{x}_{1 d}=\{0,1,0,0,0\}$, thus $\mathbf{t}_{1 d}=\{0,1,1,1,2\}$. Note that $M R T_{\text {relaxed }}$ has instructions $\left\{I_{2}, I_{3}, I_{4}\right\}$ in the same congruence class, thus it is not valid, and the validation function will return the latency $l_{1 d}=3$ and the number of conflicts $n \_$outs $=1$, resulting in fitness $1_{1 d}=3+1 * 3=6$. Furthermore, $M R T_{\text {relaxed }}$ need to be validated, before being added back to the population.

3) Validation Algorithm: Algorithm 5 describes the function used to modify non-valid $M R T \mathrm{~s}$ into valid ones, randomly handling conflicts, and also counting how many conflicts there were in the $M R T$. This function is necessary for the insemination and individual evaluation processes since invalid $M R T$ s can be generated in both procedures.

Each resource constrained instruction $I$ is attempted to be allocated in its current $M R T$ slot (lines 2 and 3), and if there is already another instruction allocated to that slot, $I$ is attempted to be scheduled in another resource instance, with the same congruence class (lines 4 to 8 ). This solves conflicts where two instructions are assigned for the same resource instance, but there are still other available instances in the same congruence class.

If all resources are busy, a randomly allocated instruction $I_{r}$ in the congruence class is selected (line 10), and the number of conflicts is incremented (line 16). Finally, both instruction dispute a coin toss to define which one will be 
allocated to a random $M R T$ empty slot (lines 11 to 15 ). This random selection is made as there is no information on which allocation is the best to be performed.

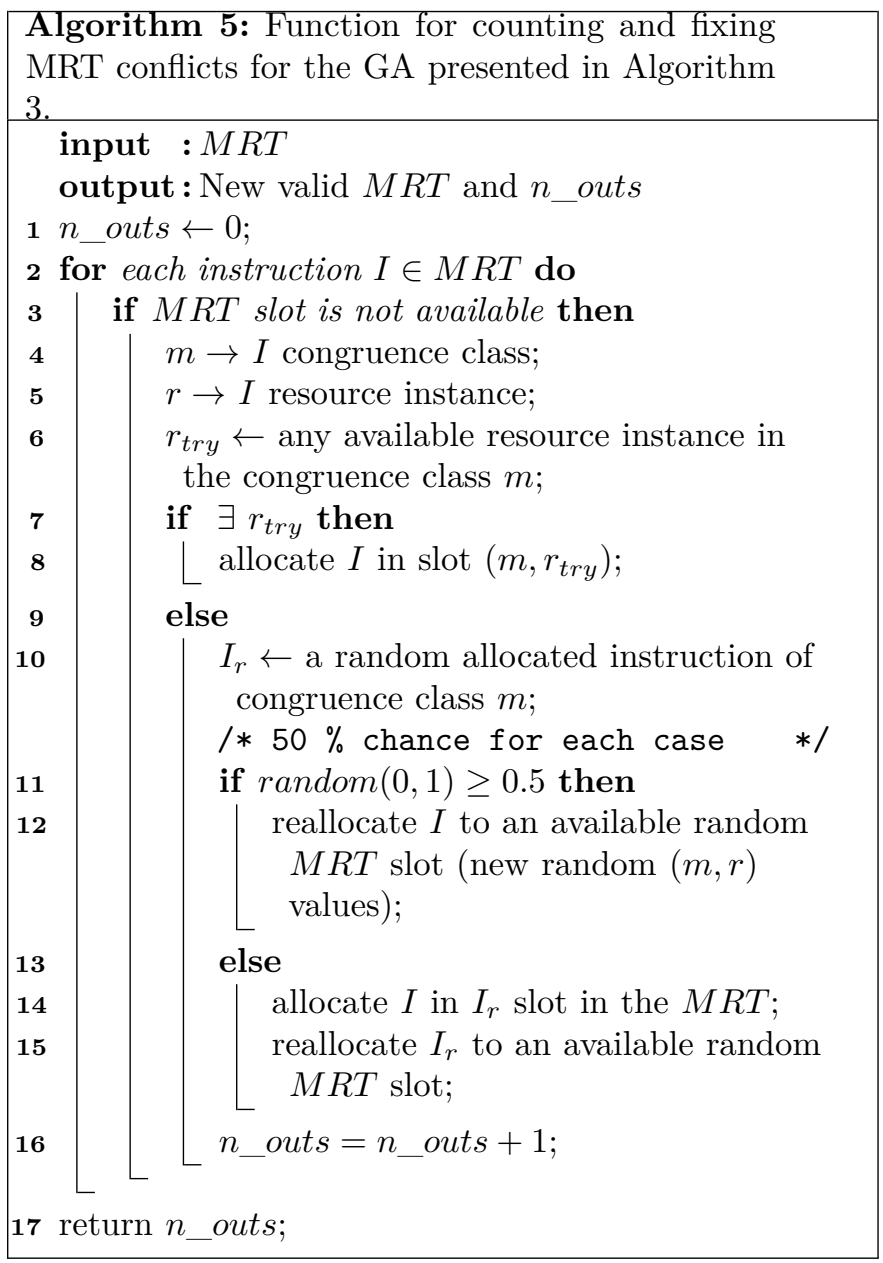

To illustrate the validation process, consider Figure 1b. First $I_{1}, I_{2}$, and $I_{3}$ will be successfully allocated in their congruence class. Then $I_{4}$ will fail to be allocated with $m_{4}=1$. A random allocated instruction with $m=1$ is chosen, which can be $I_{2}$ or $I_{3}$. Let's assume that $I_{2}$ is chosen, then a random selection between $I_{2}$ and $I_{4}$ will be performed. If $I_{2}$ is selected, it remains as it is, and $I_{4}$ is randomly allocated into an empty slot, which can result to MRTs depicted in Figure 1c or Figure 1e. If $I_{4}$ is selected, $I_{2}$ is randomly allocated which leads to the MRT depicted in Figure 1d.

4) Cross-Over Algorithm: Algorithm 6 describes the single point cross-over function used. The cross-over point is a randomly chosen number of instructions (line 1), which are copied to $c u b_{1}$ and $c u b_{2}$ respectively from parents $p_{1}$ and $p_{2}$ (lines 2 to 4 ). The rest of the instructions are copied from the opposite parent (lines 5 to 7 ). This simple process might cause MRT conflicts since an instruction of the first part of $p_{1}$ can occupy the same slot as an instruction in the second part of $p_{2}$. Possible conflicts are solved using the validation process described in Algorithm 5 .

To illustrate the cross-over, consider Figures $1 \mathrm{c}$ and $1 \mathrm{~d}$, which are represented by the lists $p_{1}=M R T_{1 c}=$

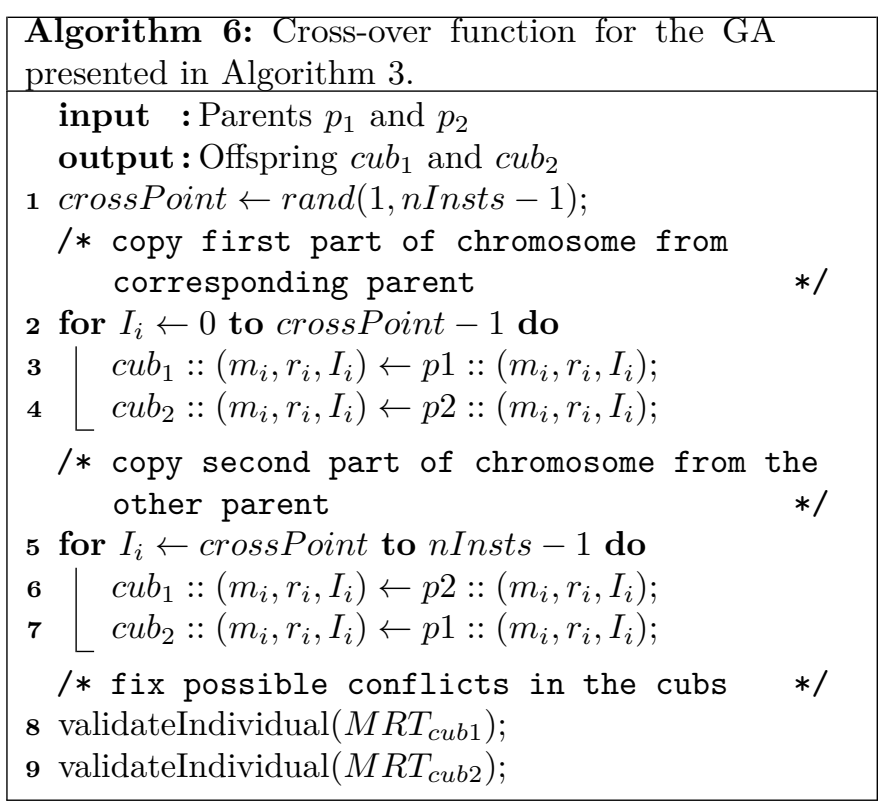

$\left\{\left(0,0, I_{1}\right),\left(1,0, I_{2}\right),\left(1,1, I_{3}\right),\left(0,1, I_{4}\right),\left(2,0, I_{5}\right)\right\}$ and $p_{2}=$ $M R T_{1 d}=\left\{\left(0,0, I_{1}\right),\left(2,0, I_{2}\right),\left(1,1, I_{3}\right),\left(1,0, I_{4}\right),\left(0,1, I_{5}\right)\right\}$ respectively. The cross-over point is a random number between 1 and 4. Supposing the cross-over point is 2 , the resulting cubs would be $c u b_{1}=$ $\left\{\left(0,0, I_{1}\right),\left(1,0, I_{2}\right),\left(1,1, I_{3}\right),\left(1,0, I_{4}\right),\left(0,1, I_{5}\right)\right\} \quad$ and $c u b_{2}=\left\{\left(0,0, I_{1}\right),\left(2,0, I_{2}\right),\left(1,1, I_{3}\right),\left(0,1, I_{4}\right),\left(2,0, I_{4}\right)\right\}$.

\section{Comparison With SDC AND ILP Modulo SCHEDULERS}

\section{A. Performance Models}

The total number of individuals evaluated by the GA is: totalIndividuals $=n P o p \times\left(1+i_{r}\right)+\operatorname{maxGen} \times$ offspringSize. Under the proposed formulation, a maximum of two SDC problems are solved for each individual. As such, for GAS to be faster than SDCS, the totalIndividuals need to be less than the number of problems solved by SDCS.

Let $n$ Pop $=$ offspringSize $=\alpha \times n$ and $\operatorname{maxGen}=\beta$, where $\alpha$ and $\beta$ are parameters defined by the user, and $n$ is the loop size. Thus, totalIndividuals $=n \times(\alpha(1+$ insemination Rate $)+\beta$ ). Since in the worst-case two SDC problems are solved per individual (Formulations 2 and 3), GAS is set to solve $\mathcal{O}(n)$ SDC problems.

The conditions for GAS to be faster than the SDCS worst-case is:

$$
\begin{aligned}
2 \times\left(\alpha n\left(1+i_{r}\right)+\beta(\alpha n)\right) & <\text { budgetRatio } \times n^{2} \\
\Rightarrow \quad 2 \alpha\left(\beta+1+i_{r}\right) 1 & <\text { budgetRatio } \times n \\
\Rightarrow \quad \frac{2 \alpha\left(\beta+1+i_{r}\right)}{\text { budgetRatio }} & <n
\end{aligned}
$$

Inequality inq. 5 provides a sufficient condition for GAS to be faster than SDCS worst-case, and indicates that there exists a large enough $n$ to which GAS will be faster than SDCS worst-case. The impact of the above condition will 
be revisited in the performance evaluation section where the parameters $\alpha$ and $\beta$ are tuned.

\section{B. Characterisation}

A high-level comparison between SDCS, ILPS and GAS is presented in Table III. The table shows that ILPS has the worst solver complexity, which also has the worst scaling with the loop size $n$.

In the case of GAS, the problem size scales better than in the case of SDCS, as GAS creates only one variable for each instruction $I_{i}$, instead of $D_{i}$ (as proposed by [10]), as well as GAS does not add constraints related to the allocation as SDCS does (Section III-A). As such, the GAS's main sources of speed-up compared to SDCS is a) the smaller number of problems solved, which is achieved due Formulation 3 usage and the efficient MRT exploration by the GA, and b) the fact that the generated problems exhibit smaller sizes.

TABLE III: Comparison between the schedulers.

\begin{tabular}{c|c|c|c}
\hline & SDCS & ILPS & GAS \\
\hline $\begin{array}{c}\text { Problem } \\
\text { type }\end{array}$ & SDC & ILP & SDC \\
\hline $\begin{array}{c}\text { Solver } \\
\text { complexity }\end{array}$ & Polynomial & Exponential & Polynomial \\
\hline Problem size & $\begin{array}{c}\text { grows during } \\
\text { execution }\end{array}$ & $\mathcal{O}\left(n^{2}\right)$ & 0 \\
\hline $\begin{array}{c}\text { Problems } \\
\text { solved }\end{array}$ & $\mathcal{O}\left(n^{2}\right)$ & 1 & $\mathcal{O}(n)$ \\
\hline$I I$ & Not optimal & Optimal & Not optimal \\
\hline Latency & Not optimal & Optimal & Not optimal \\
\hline
\end{tabular}

In case of SDCS, its main source of inefficiency lies in its iterative heuristic search for finding a solution with a valid MRT. A secondary source of inefficiency is its incapability to prove infeasibility for a given $I I$, requiring the allocated time budget expiration. Such infeasible $I I$ s are explored by [10] using a different RecMII estimation than [6], which results in smaller and infeasible $M I I$.

The ILPS's main source of inefficiency lies on the quadratic problem size scaling, and on the exponential complexity of its solver. In contrary, GAS search solves a number of problems that scale linear with $n$, as opposed to the quadratic scaling of number of problems in SDCS. Furthermore, GAS problems are in the SDC form, and thus its solver exhibits polynomial complexity.

Concerning the quality of the achieved solution, SDCS does not guarantee latency or $I I$ optimality due to its heuristic nature. If a solution cannot be found within a pre-allocated time budget, the $I I$ is increased, and a new search is performed. On the contrary, ILPS guarantees both latency and $I I$ optimality, since the solver guarantees to find the optimal solution for the problem, for a given $I I$, or declare infeasibility. GAS does not guarantee latency or II optimality since the GA may fail to find a feasible MRT before it reaches its stop criteria.

\section{Benchmark Selection}

The proposed approach is evaluated using 13 HLS benchmarks, and their details and characteristics are summarised in Table IV. All suitable loops of the above benchmarks were submitted for scheduling using the SDCS, ILPS, and GAS approaches. Benchmarks mt, dv, fat, $\mathbf{c p}$, and ac are the same ones used in [6]. Please note that benchmarks mt, fat, and ac are synthetic benchmarks that have been designed specifically to test SDCS's scalability and are originally used in [6]. These benchmarks use more than $50 \%$ resource-constrained nodes.

The rest of the benchmarks exhibit various data access pattern, loop dependencies, logic operations, nested loops, and induction variables to validate the approaches under different code constructions.

Moreover, as benchmarks rs, $\mathbf{c v}, \mathbf{g p}, \mathbf{f t}$, and $\mathbf{s h}$ contain more than one loop in their source code, the entries in Tables IV, V, and V reflect the combined loop characteristics.

All schedulers were implemented as part of LegUP [21] infrastructure, where the SDCS is natively implemented. As LegUP imposes restrictions on which loops can be pipelined, the same restrictions are also applied for ILPS and GAS. As such, all loops have bounds and array sizes modified to statically determined values, and loops with unknown bounds and more than one induction variable were set not to be pipelined. Since only innermost loops, which are in the main $\mathrm{C}$ file, can be pipelined, all benchmarks were adapted to a single $\mathrm{C}$ file, and only the innermost loops were selected to be pipelined. Furthermore, a full inline is applied in the whole code, since LegUP implements local memories only for arrays that are used exclusively within the context of one, and only one, function.

It should be noted that the above code modifications are applied due to the LegUP's restrictions. The discussed schedulers can be implemented on other compilers without these restrictions, as has been demonstrated by [10].

\section{Performance Evaluation}

The results presented in this section were obtained on an Ubuntu 14.04 machine, with 16 GB of RAM, and an Intel(R) Core(TM) i7-2600 CPU @ 3.40GHz. All schedulers are implemented inside LegUP 4.0 structure as LLVM 3.5 opt passes. Gurobi 7.5 [22] is chosen as solver for all schedulers.

The SDCS budget is set as budget Ratio $=6$ (LegUP default) and INCREMENTAL_SDC $=1$ (speeds up SDCS [23]). The ILPS time budget is set to 5 minutes.

The parameters of GAS were empirically set as follows: $\alpha=0.10, \beta=10$, and $i_{r}=0.05$, for all benchmarks, satisfying $\alpha\left(\beta+1+i_{r}\right)=1.105<6=$ budgetRatio, with the expectation that GAS will, in most cases, solve fewer problems than the SDCS worst case. The mutation rate is empirically defined as mutation Prob $=1 \%$.

The three schedulers are tested with respect to the obtained quality of solution and the time required to reach that solution on the above benchmarks. The obtained results are presented in Tables V and VI, corresponding to the average of 50 repetitions. Overall, all schedulers achieved similar results ( $I I$ and loop latency), but with a clear time improvement for the proposed GAS scheduler. 
TABLE IV: Benchmark selection.

\begin{tabular}{|c|c|c|c|c|c|c|c|c|}
\hline Name & $\begin{array}{l}\text { Short } \\
\text { Name }\end{array}$ & Benchmark & $\begin{array}{l}\text { Loop } \\
\text { size }\end{array}$ & $\begin{array}{l}\text { Ar- } \\
\text { rays } \\
\text { Size }\end{array}$ & $\begin{array}{c}\text { Operations } \\
+/ / \% / \mathrm{f}+/ \mathrm{f}^{*} / \mathrm{f} \% /[]\end{array}$ & $\begin{array}{c}\text { Constraints } \\
+/{ }^{*} / \% / \mathrm{f}+/ \mathrm{f}^{*} / \mathrm{f} \% /[]\end{array}$ & $\begin{array}{c}\text { Dis- } \\
\text { tance }\end{array}$ & $\begin{array}{l}\text { recMII// } \\
\text { resMII/ } \\
\text { MII }\end{array}$ \\
\hline rs decoder [15] & rs & none & 7 & 256 & $0 / 1 / 0 / 0 / 0 / 0 / 18$ & $\mathrm{X} / 1 / \mathrm{X} / \mathrm{X} / \mathrm{X} / \mathrm{X} / 2$ & 1 & $8 / 13 / 13$ \\
\hline covariance $[16]$ & $\mathrm{cV}$ & Polybench & 38 & 500 & $0 / 0 / 0 / 1 / 1 / 0 / 6$ & $\mathrm{X} / \mathrm{X} / \mathrm{X} / 1 / 1 / \mathrm{X} / 2$ & 1 & $47 / 4 / 47$ \\
\hline gauss pivoting [17] & gp & MIT [18] & 45 & 100 & $0 / 0 / 0 / 2 / 1 / 0 / 8$ & $\mathrm{X} / \mathrm{X} / \mathrm{X} / 1 / 1 / \mathrm{X} / 2$ & 1 & $23 / 5 / 23$ \\
\hline row col $[17]$ & $\mathrm{rc}$ & Xilinx & 18 & 100 & $0 / 0 / 0 / 0 / 0 / 0 / 6$ & $\mathrm{X} / \mathrm{X} / \mathrm{X} / \mathrm{X} / \mathrm{X} / \mathrm{X} / 2$ & 200 & $1 / 3 / 3$ \\
\hline fft [19] & $\mathrm{ft}$ & LegUp & 55 & 1024 & $0 / 0 / 1 / 0 / 0 / 0 / 6$ & $\mathrm{X} / \mathrm{X} / 1 / \mathrm{X} / \mathrm{X} / \mathrm{X} / 2$ & 1 & $3 / 5 / 5$ \\
\hline sha $[20],[10]$ & sh & CHStone & 111 & 8192 & $0 / 0 / 0 / 0 / 0 / 0 / 4$ & $\mathrm{X} / \mathrm{X} / \mathrm{X} / \mathrm{X} / \mathrm{X} / \mathrm{X} / 2$ & 1 & $5 / 9 / 9$ \\
\hline jacobi 2d [17] & $\mathrm{j} 2$ & Polybench & 28 & 100 & $0 / 0 / 0 / 4 / 1 / 0 / 6$ & $\mathrm{X} / \mathrm{X} / \mathrm{X} / 3 / 1 / \mathrm{X} / 2$ & 1 & $75 / 3 / 75$ \\
\hline multipliers [6] & $\mathrm{mt}$ & LegUp & 28 & 100 & $7 / 2 / 0 / 0 / 0 / 0 / 7$ & $3 / 1 / \mathrm{X} / \mathrm{X} / \mathrm{X} / \mathrm{X} / 1$ & 1 & $3 / 4 / 4$ \\
\hline dividers [6] & $\mathrm{dv}$ & LegUp & 72 & 100 & $12 / 0 / 4 / 0 / 0 / 0 / 11$ & $3 / \mathrm{X} / 3 / \mathrm{X} / \mathrm{X} / \mathrm{X} / 2$ & 1 & $3 / 6 / 6$ \\
\hline faddtree [6] & fat & LegUp & 81 & 100 & $0 / 0 / 0 / 21 / 0 / 0 / 22$ & $\mathrm{X} / \mathrm{X} / \mathrm{X} / 3 / \mathrm{X} / \mathrm{X} / 2$ & 1 & $27 / 11 / 27$ \\
\hline add int [17] & ai & LivemoreC & 82 & 100 & $0 / 0 / 0 / 21 / 9 / 0 / 12$ & $\mathrm{X} / \mathrm{X} / \mathrm{X} / 3 / 3 / \mathrm{X} / 2$ & 1 & $1 / 12 / 12$ \\
\hline complex [6] & ср & LegUp & 98 & 100 & $0 / 7 / 2 / 0 / 0 / 0 / 25$ & $\mathrm{X} / 3 / 1 / \mathrm{X} / \mathrm{X} / \mathrm{X} / 2$ & 9 & $20 / 13 / 20$ \\
\hline adderchain [6] & $\mathrm{ac}$ & LegUp & 92 & 100 & $48 / 0 / 0 / 0 / 0 / 0 / 24$ & $3 / \mathrm{X} / \mathrm{X} / \mathrm{X} / \mathrm{X} / \mathrm{X} / 2$ & 1 & $3 / 12 / 12$ \\
\hline
\end{tabular}

TABLE V: Number and sizes of problems solved.

\begin{tabular}{|c|c|c|c|c|c|c|c|c|c|}
\hline \multirow[t]{2}{*}{ Name } & \multicolumn{3}{|c|}{ \# Solves } & \multicolumn{3}{|c|}{ \# Variables } & \multicolumn{3}{|c|}{ \# Constraints } \\
\hline & SDCS & ILPS & GAS & SDCS & ILPS & GAS & SDCS & ILPS & GAS \\
\hline rs & 50 & 8 & 48 & 119 & 344 & 84 & 341 & 816 & 82 \\
\hline $\mathrm{cV}$ & 22 & 3 & 57.80 & 107 & 131 & 38 & 324 & 264 & 43 \\
\hline gp & 24 & 3 & 50.93 & 103 & 177 & 45 & 326 & 418 & 66 \\
\hline $\mathrm{rc}$ & 13 & 1 & 6 & 28 & 117 & 18 & 105 & 332 & 35 \\
\hline $\mathrm{ft}$ & 25 & 3 & 18 & 75 & 201 & 55 & 217 & 480 & 68 \\
\hline sh & 57 & 5 & 85.23 & 125 & 425 & 111 & 347 & 1071 & 137 \\
\hline $\mathrm{j} 2$ & 25 & 1 & 21.70 & 112 & 229 & 28 & 365 & 638 & 39 \\
\hline $\mathrm{mt}$ & 23 & 1 & 19.80 & 78 & 208 & 28 & 262 & 584 & 42 \\
\hline $\mathrm{dv}$ & 67 & 1 & 66.13 & 351 & 534 & 73 & 1143 & 1622 & 157 \\
\hline fat & 175 & 1 & 84.67 & 402 & 2113 & 81 & 1333 & 6929 & 103 \\
\hline ai & 94 & 1 & 62 & 530 & 1114 & 82 & 1694 & 3387 & 105 \\
\hline $\mathrm{cp}$ & 373 & 1 & 120.17 & 399 & 1488 & 98 & 1291 & 4837 & 159 \\
\hline $\mathrm{ac}$ & 470 & 1 & 96.60 & 145 & 1276 & 100 & 569 & 4133 & 149 \\
\hline geomean & 56.09 & 1.71 & 43.90 & 145.43 & 409.35 & 56.43 & 467.98 & 1119.88 & 79.42 \\
\hline ratio & 1 & 0.03 & 0.78 & 1 & 2.81 & 0.39 & 1 & 2.39 & 0.17 \\
\hline
\end{tabular}

Table $\mathrm{V}$ presents the number of problems solved, and the number of variables and constraints for all schedulers. As the results demonstrate, GAS solves significantly simpler problems (i.e. fewer variables and constraints) than SDCS. Furthermore, the larger (i.e. more instructions) the code is, the fewer problems are solved by GAS when compared to SDCS.

Table VI presents the obtained $I I$, the solution's latency, the total number of cycles, and the scheduling time to reach the solution, for all loops of all benchmarks. Results show that all schedulers reached the minimum $I I$.

With respect to the obtained solution latency, SDCS produce solutions with $12 \%$ longer latency when compared to ILPS, while GAS produces solutions with $13 \%$ longer latency when compared with SDCS. However, the impact of the latency is amortized in the total number of cycles in the equation $t_{\text {total }}$ cycles $=l+I I \times(t c-1)$, as $t c$ usually is a larger number than $l$. As illustrated in Table VI, the solutions produced by GAS exhibit on average $1 \%$ penalty in the total cycles.

Finally, Table VI shows the computation time taken by SDCS, ILPS and GAS. ILPS is not comparable to the other approaches due to its exponential scaling with the problem size. GAS presents $55 \%$ improvement over SDCS in our tests (geomean).

To exemplify scalability, Figures 5 and 6 present the computation time and achieved $I I$ as a function of the loop size, for the benchmarks in Table IV when loop unrolling is applied. The unrolling factor is presented in the number above the GAS marks. Benchmarks $\mathbf{g p}, \mathbf{f t}, \mathbf{s h}$, and $\mathbf{j} \mathbf{2}$ are excluded from this evaluation due to small unrolling factors that they can support (i.e. their loops can be unrolled only by factors 1 and 2 ).

Figure 5 illustrates that the proposed GAS approach scales better with the loop size than SDCS. Moreover, the results show that GAS can provide solutions for long codes, where for the same cases SDCS fails (missing points).

Moreover, Figure 6 shows that GAS can find smaller $I I \mathrm{~s}$ than SDCS in the case of long codes, which indicates an improved performance on the total number of cycles for large codes, contrasting the results obtained for smaller codes presented on Table VI.

Figure 7 shows the GAS solution latency for the benchmark $\mathbf{c p}$ (the most challenging benchmark as indicated by Tables V and VI) as a function of the number of generations and parameterized according to the population size. Figure 7 shows how the population size and number of generations can be increased to obtain a smaller latency, showing the 
TABLE VI: Performance and computation time results.

\begin{tabular}{|c|c|c|c|c|c|c|c|c|c|c|c|c|}
\hline \multirow[t]{2}{*}{ Name } & \multicolumn{3}{|c|}{ II } & \multicolumn{3}{|c|}{ Latency } & \multicolumn{3}{|c|}{ Total Cycles } & \multicolumn{3}{|c|}{ Scheduling Time (s) } \\
\hline & SDCS & ILPS & GAS & SDCS & ILPS & GAS & SDCS & ILPS & GAS & SDCS & ILPS & GAS \\
\hline rs & 13 & 13 & 13 & 23 & 18 & $\begin{array}{l}20.63 \\
\pm 0.74\end{array}$ & 897 & 892 & $\begin{array}{l}894.63 \\
\pm 0.74\end{array}$ & $\begin{array}{c}1.04 \mathrm{e}-02 \\
\pm 1.02 \mathrm{e}-03\end{array}$ & $\begin{array}{c}3.61 \mathrm{e}-01 \\
\pm 2.21 \mathrm{e}-02\end{array}$ & $\begin{array}{c}8.47 \mathrm{e}-03 \\
\pm 8.30 \mathrm{e}-04\end{array}$ \\
\hline $\mathrm{cV}$ & 47 & 47 & 47 & 65 & 62 & $62 \pm 0$ & 2368 & 2365 & $\begin{array}{c}2365 \\
\pm 0\end{array}$ & $\begin{array}{c}5.70 \mathrm{e}-03 \\
\pm 5.56 \mathrm{e}-04\end{array}$ & $\begin{array}{c}1.05 \mathrm{e}-02 \\
\pm 1.32 \mathrm{e}-03\end{array}$ & $\begin{array}{c}8.51 \mathrm{e}-03 \\
\pm 1.34 \mathrm{e}-03\end{array}$ \\
\hline gp & 23 & 23 & 23 & 53 & 49 & $49 \pm 0$ & 30 & 26 & $26 \pm 0$ & $\begin{array}{c}6.94 \mathrm{e}-03 \\
\pm 7.24 \mathrm{e}-04\end{array}$ & $\begin{array}{c}1.77 \mathrm{e}-02 \\
\pm 1.41 \mathrm{e}-03\end{array}$ & $\begin{array}{c}8.96 \mathrm{e}-03 \\
\pm 9.38 \mathrm{e}-04\end{array}$ \\
\hline $\mathrm{rc}$ & 3 & 3 & 3 & 6 & 6 & $\begin{array}{c}6.43 \\
\pm 0.34\end{array}$ & 303 & 303 & $\begin{array}{c}303.43 \\
\pm 0.34\end{array}$ & $\begin{array}{c}3.70 \mathrm{e}-03 \\
\pm 4.60 \mathrm{e}-04\end{array}$ & $\begin{array}{c}6.95 \mathrm{e}-02 \\
\pm 4.29 \mathrm{e}-03\end{array}$ & $\begin{array}{c}1.45 \mathrm{e}-03 \\
\pm 1.40 \mathrm{e}-04\end{array}$ \\
\hline $\mathrm{ft}$ & 5 & 5 & 5 & 13 & 13 & $\begin{array}{l}14.50 \\
\pm 0.61\end{array}$ & 648 & 648 & $\begin{array}{l}649.50 \\
\pm 0.61\end{array}$ & $\begin{array}{c}5.18 \mathrm{e}-03 \\
\pm 2.34 \mathrm{e}-04\end{array}$ & $\begin{array}{c}1.49 \mathrm{e}-01 \\
\pm 1.09 \mathrm{e}-02\end{array}$ & $\begin{array}{c}3.50 \mathrm{e}-03 \\
\pm 2.52 \mathrm{e}-04\end{array}$ \\
\hline sh & 9 & 9 & 9 & 13 & 9 & $\begin{array}{l}12.37 \\
\pm 0.88\end{array}$ & 177 & 173 & $\begin{array}{l}176.37 \\
\pm 0.88\end{array}$ & $\begin{array}{c}1.39 \mathrm{e}-02 \\
\pm 1.11 \mathrm{e}-03\end{array}$ & $\begin{array}{c}3.47 \mathrm{e}-01 \\
\pm 1.18 \mathrm{e}-02\end{array}$ & $\begin{array}{c}1.84 \mathrm{e}-02 \\
\pm 7.50 \mathrm{e}-04\end{array}$ \\
\hline $\mathrm{j} 2$ & 75 & 75 & 75 & 76 & 75 & $\begin{array}{c}139.50 \\
\pm 7.80\end{array}$ & 7351 & 7350 & $\begin{array}{c}7414.50 \\
\pm 7.80\end{array}$ & $\begin{array}{c}1.12 \mathrm{e}-02 \\
\pm 5.32 \mathrm{e}-04\end{array}$ & $\begin{array}{c}8.25 \mathrm{e}-01 \\
\pm 2.43 \mathrm{e}-02\end{array}$ & $\begin{array}{c}5.23 \mathrm{e}-03 \\
\pm 6.95 \mathrm{e}-04\end{array}$ \\
\hline $\mathrm{mt}$ & 4 & 4 & 4 & 24 & 23 & $\begin{array}{l}27.60 \\
\pm 1.31\end{array}$ & 380 & 379 & $\begin{array}{l}383.60 \\
\pm 1.31\end{array}$ & $\begin{array}{c}8.54 \mathrm{e}-03 \\
\pm 5.39 \mathrm{e}-04\end{array}$ & $\begin{array}{c}1.66 \mathrm{e}+00 \\
\pm 4.59 \mathrm{e}-02\end{array}$ & $\begin{array}{c}3.88 \mathrm{e}-03 \\
\pm 4.36 \mathrm{e}-04\end{array}$ \\
\hline $\mathrm{dv}$ & 6 & 6 & 6 & 72 & 71 & $\begin{array}{l}78.20 \\
\pm 1.67\end{array}$ & 306 & 305 & $\begin{array}{l}312.20 \\
\pm 1.67\end{array}$ & $\begin{array}{c}6.04 \mathrm{e}-02 \\
\pm 8.51 \mathrm{e}-05\end{array}$ & $\begin{array}{l}3.32 \mathrm{e}+02 \\
\pm 5.28 \mathrm{e}-01\end{array}$ & $\begin{array}{c}2.54 \mathrm{e}-02 \\
\pm 3.19 \mathrm{e}-03\end{array}$ \\
\hline fat & 27 & 27 & 27 & 101 & 101 & $\begin{array}{c}140.23 \\
\pm 7.63\end{array}$ & 1154 & 1154 & $\begin{array}{c}1193.23 \\
\pm 7.63\end{array}$ & $\begin{array}{c}1.67 \mathrm{e}-01 \\
\pm 2.34 \mathrm{e}-03\end{array}$ & $\begin{array}{c}1.19 \mathrm{e}+03 \\
\pm 5.30 \mathrm{e}-01\end{array}$ & $\begin{array}{c}2.79 \mathrm{e}-02 \\
\pm 2.46 \mathrm{e}-03\end{array}$ \\
\hline ai & 12 & 12 & 12 & 95 & 91 & $\begin{array}{r}136.80 \\
\pm 3.34\end{array}$ & 191 & 187 & $\begin{array}{c}232.80 \\
\pm 3.34\end{array}$ & $\begin{array}{c}1.31 \mathrm{e}-01 \\
\pm 2 \mathrm{e}-03\end{array}$ & $\begin{array}{l}1.15 \mathrm{e}+02 \\
\pm 2.19 \mathrm{e}-01\end{array}$ & $\begin{array}{c}2.01 \mathrm{e}-02 \\
\pm 2.17 \mathrm{e}-03\end{array}$ \\
\hline $\mathrm{cp}$ & 20 & 20 & 20 & 127 & 122 & $\begin{array}{l}141.37 \\
\pm 4.20\end{array}$ & 1807 & 1802 & $\begin{array}{c}1821.37 \\
\pm 4.20\end{array}$ & $\begin{array}{c}3.54 \mathrm{e}-01 \\
\pm 3.57 \mathrm{e}-03\end{array}$ & $\begin{array}{l}1.19 \mathrm{e}+03 \\
\pm 3.23 \mathrm{e}-01\end{array}$ & $\begin{array}{c}5.61 \mathrm{e}-02 \\
\pm 5.69 \mathrm{e}-03\end{array}$ \\
\hline $\mathrm{ac}$ & 12 & 12 & 12 & 25 & 12 & $\begin{array}{l}27.33 \\
\pm 2.10\end{array}$ & 1093 & 1080 & $\begin{array}{c}1095.33 \\
\pm 2.10\end{array}$ & $\begin{array}{c}2.36 \mathrm{e}-01 \\
\pm 5.24 \mathrm{e}-03\end{array}$ & $\begin{array}{l}1.19 \mathrm{e}+03 \\
\pm 2.76 \mathrm{e}-01\end{array}$ & $\begin{array}{c}3.57 \mathrm{e}-02 \\
\pm 2.20 \mathrm{e}-03\end{array}$ \\
\hline geomean & 12.87 & 12.87 & 12.87 & 37.80 & 33.36 & 42.79 & 579.12 & 569.63 & 585.05 & $2.45 \mathrm{e}-02$ & $3.66 \mathrm{e}+00$ & $1.10 \mathrm{e}-02$ \\
\hline ratio & 1 & 1 & 1 & 1 & 0.88 & 1.13 & 1 & 0.98 & 1.01 & 1 & 149 & 0.45 \\
\hline
\end{tabular}
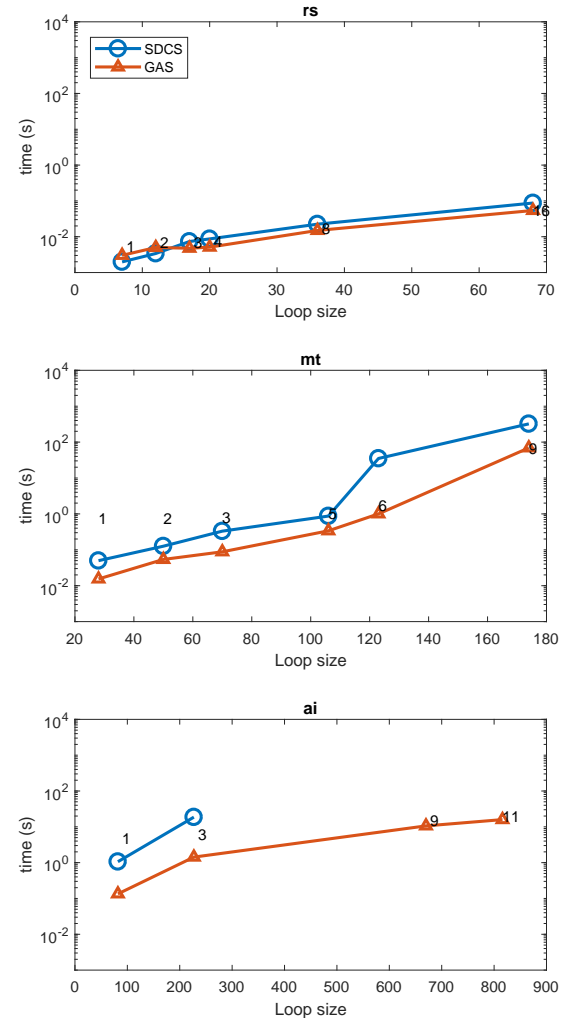
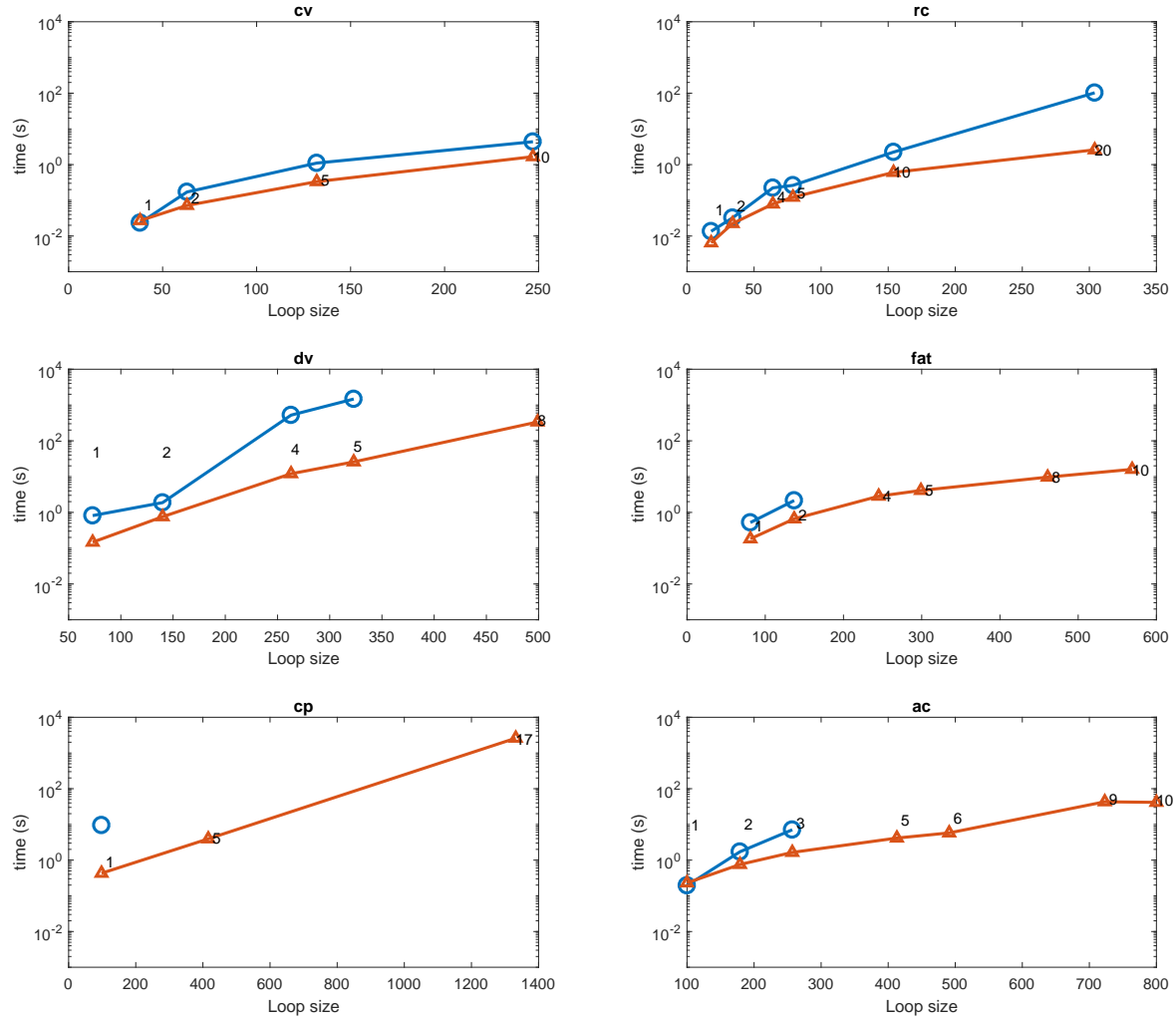

Fig. 5: Scheduling time scaling as a function of the loop unrolling factor. 

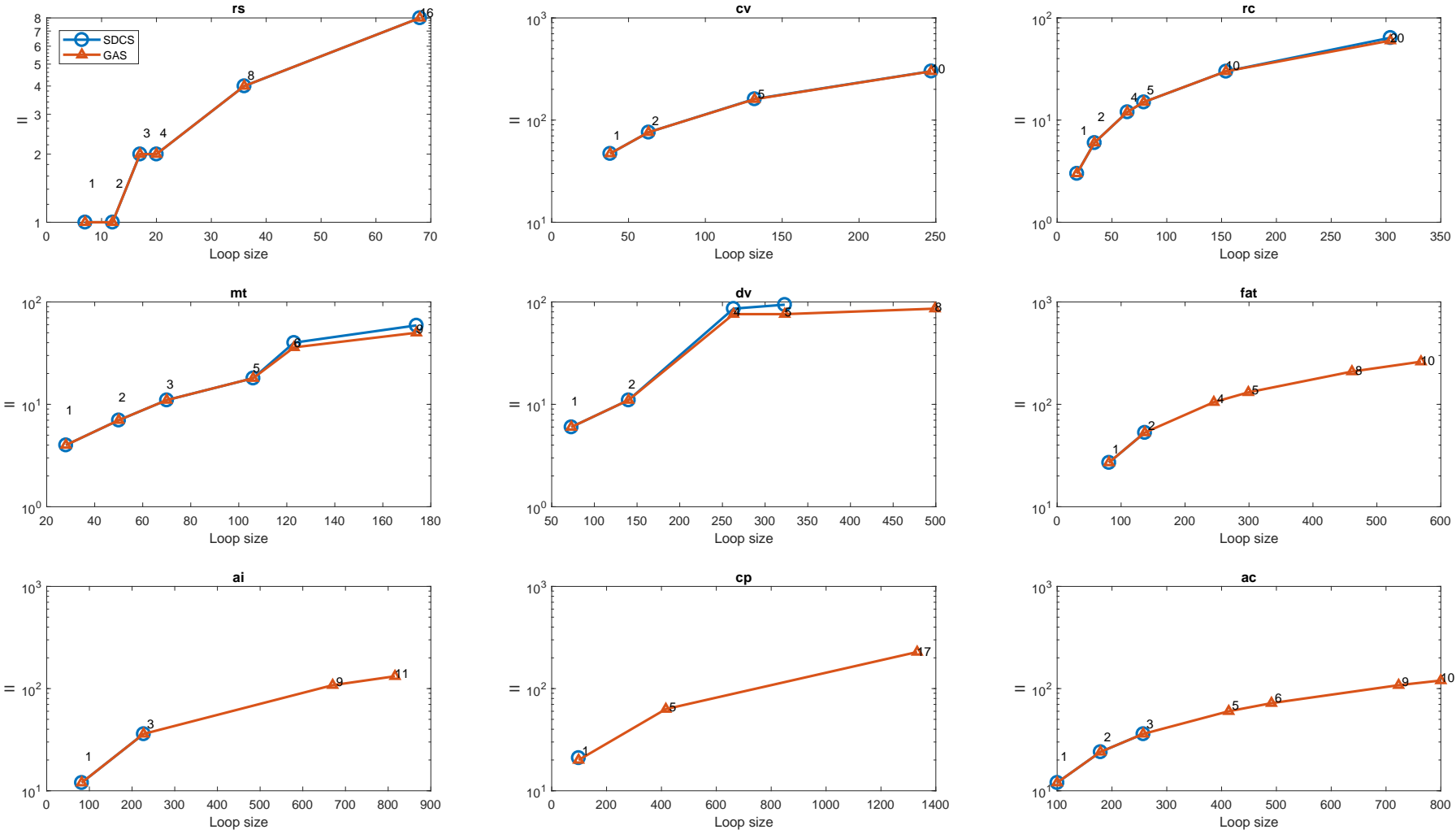

Fig. 6: Final II scaling as a function of the loop unrolling factor.

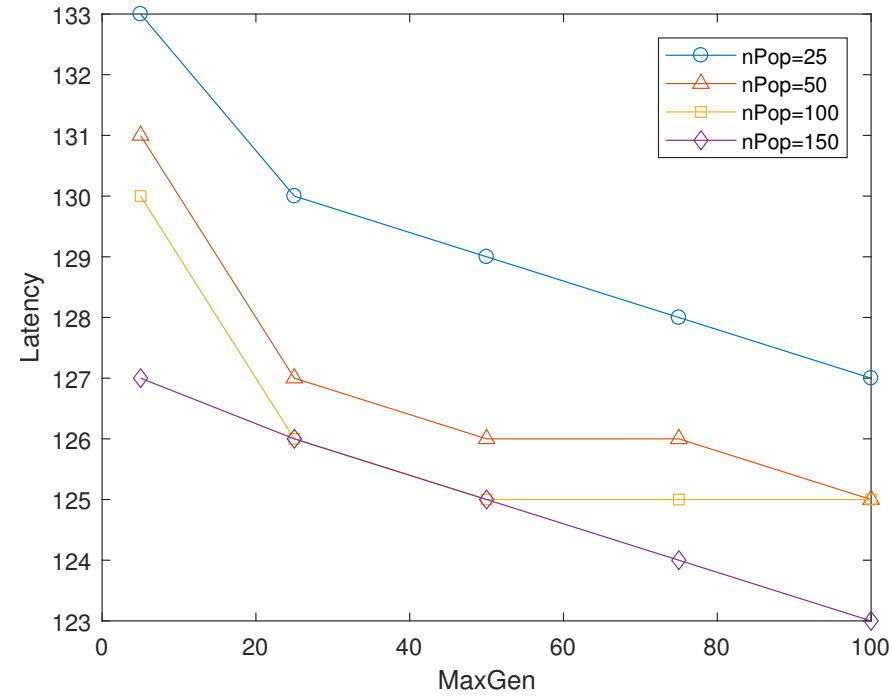

Fig. 7: Latency evolution according to the number of generations for benchmark cp.

trade-off between computation time and solution quality.

It should be noted that the results reported are obtained by applying the above module scheduling approach on a small number of loops. However, in the case of a design space exploration in a large application, multiple kernels need to be considered for loop pipelining, resulting in a combinatorial number of possible configurations. As such the obtained gains from the proposed approach will reflect to a significant reduction of the required absolute design space exploration time. For example, the DSE method presented by [5] evaluates up to 250 designs per benchmark. Some of these evaluations vary the loops unrolling factor (which is equivalent to our scaling tests presented on Figures 5 and 6).

Finally, tests were performed in order to evaluate the performance of the methods in cases where the initial minimum $I I, M I I$, is underestimated, leading to cases of infeasible $I I \mathrm{~s}$. As such, benchmarks with RecMII > ResMII are selected and the MII is set to ResMII in order to force the schedulers to handle cases where the candidate $I I$ is infeasible. The obtained results, presented in Table VII, show that all schedulers spend more time to provide a solution as compared to Table VI, since now they also handle cases where $I I$ is infeasible, however, the schedulers relative performance has not changed.

TABLE VII: Computation time (s) for $M I I=\operatorname{ResII}$.

\begin{tabular}{|c|c|c|c|c|c|c|c|}
\hline Name & cv & gp & j2 & fat & cp & geom. & ratio \\
\hline SDCS & 0.02 & 0.02 & 0.04 & 0.18 & 0.36 & 0.06 & 1 \\
\hline ILPS & 0.14 & 0.15 & 2.18 & $1,12 \mathrm{e} 3$ & $1.17 \mathrm{e} 3$ & 9.04 & 142.17 \\
\hline GAS & 0.01 & 0.01 & 0.02 & 0.03 & 0.05 & 0.02 & 0.31 \\
\hline
\end{tabular}

\section{Conclusion}

The work in this paper presents a novel formulation for the Modulo Scheduling problem, as well as a proposed solution that is based partially on the use of a Genetic Algorithm. Results have shown that a significant speedup can be achieved compared to the current state-of-the-art 
approaches, where the proposed formulation also enjoys better scalability properties paving the way for attacking larger source codes.

In summary, the results show that both ILPS and GAS can produce valid schedules, which are not optimal if a limited time budget is enforced. The ILPS produces nonoptimal solutions during the branch and bound process in the solver, and GAS produces a valid non-"proven to be"-optimal solution for each individual. Furthermore, the resulting non-minimum latency contributes weakly to the total number of cycles for the complete loop execution, what minimizes the GAS impact on the overall loop performance.

The obtained results illustrate that the proposed scheduler achieves the number of SDC problems solved $\mathcal{O}(n)$ scalability, where $n$ is the number of LLVM IR instructions in the loop code. This is a significant improvement compared to the worst-case scalability $\mathcal{O}\left(n^{2}\right)$ provided by the current state-of-the-art SDCS approach.

Furthermore, the scaling test results indicate that GAS is capable of finding solutions for large codes where SDCS fails to do so, and also suggest that GAS is capable to find smaller $I I$ than SCDS, indicating a more efficient exploration of the solution space.

\section{ACKNOWLEDGMENT}

The authors would like to thank the São Paulo Research Fundation (FAPESP) for the financial support given to this project (grant number 2016/13327-6).

\section{REFERENCES}

[1] B. C. Schafer and K. Wakabayashi, "Divide and conquer high-level synthesis design space exploration," ACM Trans. Des. Autom. Electron. Syst., vol. 17, no. 3, pp. 29:1-29:19, Jul. 2012. [Online]. Available: http://doi.acm.org/10.1145/2209291. 2209302

[2] Z. Wang, B. He, W. Zhang, and S. Jiang, "A performance analysis framework for optimizing OpenCL applications on FPGAs," in 2016 IEEE International Symposium on High Performance Computer Architecture (HPCA), March 2016, pp. 114-125.

[3] B. C. Schafer, T. Takenaka, and K. Wakabayashi, "Adaptive simulated annealer for high-level synthesis design space exploration," in 2009 International Symposium on VLSI Design, Automation and Test, April 2009, pp. 106-109.

[4] L.-N. Pouchet, P. Zhang, P. Sadayappan, and J. Cong, "Polyhedral-based data reuse optimization for configurable computing," in Proceedings of the ACM/SIGDA International Symposium on Field Programmable Gate Arrays, ser. FPGA '13. New York, NY, USA: ACM, 2013, pp. 29-38. [Online]. Available: http://doi.acm.org/10.1145/2435264.2435273

[5] B. C. Schafer, "Probabilistic multiknob high-level synthesis design space exploration acceleration," IEEE Transactions on Computer-Aided Design of Integrated Circuits and Systems, vol. 35, no. 3, pp. 394-406, March 2016.

[6] A. Canis, S. D. Brown, and J. H. Anderson, "Modulo SDC scheduling with recurrence minimization in high-level synthesis," in 201424 th International Conference on Field Programmable Logic and Applications (FPL), Sept 2014, pp. 1-8.

[7] Z. Zhang and B. Liu, "SDC-based modulo scheduling for pipeline synthesis," in Proceedings of the International Conference on Computer-Aided Design, ser. ICCAD '13. Piscataway, NJ, USA: IEEE Press, 2013, pp. 211-218. [Online]. Available: http://dl.acm.org/citation.cfm?id=2561828.2561872

[8] J. Cong and Z. Zhang, "An efficient and versatile scheduling algorithm based on SDC formulation," in $200643 \mathrm{rd} A C M / I E E E$ Design Automation Conference, July 2006, pp. 433-438.
[9] J. Cong, B. Liu, and Z. Zhang, "Scheduling with soft constraints," in Proceedings of the 2009 International Conference on Computer-Aided Design, ser. ICCAD '09. New York, NY, USA: ACM, 2009, pp. 47-54. [Online]. Available: http://doi.acm.org/10.1145/1687399.1687410

[10] J. Oppermann, A. Koch, M. Reuter-Oppermann, and O. Sinnen, "ILP-based modulo scheduling for high-level synthesis," in Proceedings of the International Conference on Compilers, Architectures and Synthesis for Embedded Systems, ser. CASES '16. New York, NY, USA: ACM, 2016, pp. 1:1-1:10. [Online]. Available: http://doi.acm.org/10.1145/2968455.2968512

[11] S. Venugopalan and O. Sinnen, "ILP formulations for optimal task scheduling with communication delays on parallel systems," IEEE Transactions on Parallel and Distributed Systems, vol. 26, no. 1, pp. 142-151, Jan 2015.

[12] J. M. Codina, J. Llosa, and A. González, "A comparative study of modulo scheduling techniques," in Proceedings of the 16th International Conference on Supercomputing, ser. ICS '02. New York, NY, USA: ACM, 2002, pp. 97-106. [Online]. Available: http://doi.acm.org/10.1145/514191.514208

[13] M. Mitchell, "An introduction to genetic algorithms," 1996.

[14] L. Rosa, A. Delbem, C. Toledo, and V. Bonato, "Design and analysis of evolutionary bit-length optimization algorithms for floating to fixed-point conversion," Applied Soft Computing, vol. 49, pp. 447 - 461, 2016. [Online]. Available: http://www. sciencedirect.com/science/article/pii/S156849461630429X

[15] A. Agarwal, M. C. Ng, and Arvind, "A comparative evaluation of high-Level hardware synthesis using reed solomon decoder," IEEE Embedded Systems Letters, vol. 2, no. 3, pp. 72-76, Sept 2010.

[16] W. Zuo, W. Kemmerer, J. B. Lim, L.-N. Pouchet, A. Ayupov, T. Kim, K. Han, and D. Chen, "A polyhedral-based systemc modeling and generation framework for effective low-power design space exploration," in Proceedings of the IEEE/ACM International Conference on ComputerAided Design, ser. ICCAD '15. Piscataway, NJ, USA: IEEE Press, 2015, pp. 357-364. [Online]. Available: http: //dl.acm.org/citation.cfm?id $=2840819.2840870$

[17] J. Liu, S. Bayliss, and G. A. Constantinides, "Offline synthesis of online dependence testing: parametric loop pipelining for HLS," in 2015 IEEE 23rd Annual International Symposium on Field-Programmable Custom Computing Machines, May 2015, pp. 159-162.

[18] "Gaussian elimination with pivoting." [Online]. Available: http: //web.mit.edu/10.001/Web/CourseNotes/GaussPivoting.c

[19] A. Canis, J. Choi, M. Aldham, V. Zhang, A. Kammoona, J. H Anderson, S. Brown, and T. Czajkowski, "LegUp: high-level synthesis for FPGA-based processor/accelerator systems," in Proceedings of the 19th ACM/SIGDA International Symposium on Field Programmable Gate Arrays, ser. FPGA '11. New York, NY, USA: ACM, 2011, pp. 33-36. [Online]. Available: http://doi.acm.org/10.1145/1950413.1950423

[20] A. Prost-Boucle, O. Muller, and F. Rousseau, "Fast and standalone design space exploration for high-level synthesis under resource constraints," Journal of Systems Architecture, vol. 60, no. 1, pp. 79 - 93, 2014. [Online]. Available: http:// www.sciencedirect.com/science/article/pii/S1383762113001938

[21] A. Canis, J. Choi, M. Aldham, V. Zhang, A. Kammoona, T. Czajkowski, S. D. Brown, and J. H. Anderson, "LegUp: an open-source high-level synthesis tool for FPGA-based processor/accelerator systems," ACM Trans. Embed. Comput. Syst., vol. 13, no. 2, pp. 24:1-24:27, Sep. 2013. [Online]. Available: http://doi.acm.org/10.1145/2514740

[22] G. Optimization, "Inc.,"gurobi optimizer reference manual," 2017," URL: http://www.gurobi.com, 2017.

[23] A. Canis, J. Choi, B. Fort, R. Lian, Q. Huang, N. Calagar, M. Gort, J. J. Qin, M. Aldham, T. Czajkowski, S. Brown, and J. Anderson, "From software to accelerators with legUp high-level synthesis," in Proceedings of the 2013 International Conference on Compilers, Architectures and Synthesis for Embedded Systems, ser. CASES '13. Piscataway, NJ, USA: IEEE Press, 2013, pp. 18:1-18:9. [Online]. Available: http://dl.acm.org/citation.cfm?id=2555729.2555747 
The authors would like to thank the reviewers for their valuable comments and feedbackthat let to the improvement of this paper. In the newly submitted version, we have addressed all requests, and the details are given below.

\section{REVIEWER \# 1}

The author defines the loop size is the number of instructions, but the problem formulation for ILP in TABLE II, the $\mathrm{n}$ represents the operations number. Because the instructions usually have more than one operations in loops. So they are not the same.

We are sorry for the nomenclature conflict. While [10] uses operations, we use IR instructions, but both refer to the same thing, an operation or instruction executed on a functional unit. We corrected the nomenclature in the text.

Though the problem size $(\mathrm{O}(\mathrm{n}))$ can scale with the loop size as announced in the proposed method, we think the author should give a detailed complexity analysis when adding the GA method, rather than just simply give the conclusion of polynomial complexity (TABLE III). Only the algorithm complexity doesn't grow too large or can hold a similar polynomial complexity after adding the GA method, then the reduction in problem size can bring benefits.

Section VI-A presents as analysis on how many individuals (thus MRTs and SDCs problems solved) the GAS is set to solve. We updated the second paragraph to reflect the idea more clearly.

\section{REVIEWER \# 2}

We thank you for the valuable comments and observations. 